\title{
Critical exponent of Fujita-type for the semilinear damped wave equation on the Heisenberg group with power nonlinearity
}

\author{
Vladimir Georgiev ${ }^{\mathrm{a}, \mathrm{b}, \mathrm{c}}$, Alessandro Palmieri ${ }^{\mathrm{a}}$ \\ ${ }^{a}$ Department of Mathematics, University of Pisa, Largo B. Pontecorvo 5, 56127 Pisa, Italy \\ ${ }^{b}$ Faculty of Science and Engineering, Waseda University 3-4-1, Okubo, Shinjuku-ku, Tokyo 169-8555, Japan \\ ${ }^{c}$ Institute of Mathematics and Informatics-BAS Acad. G. Bonchev Str., Block 8, 1113 Sofia, Bulgaria
}

\begin{abstract}
In this paper, we consider the Cauchy problem for the semilinear damped wave equation on the Heisenberg group with power non-linearity. We prove that the critical exponent is the Fujita exponent $p_{\text {Fuj }}(\mathbb{Q})=1+2 / \mathbb{Q}$, where $\mathbb{Q}$ is the homogeneous dimension of the Heisenberg group.

On the one hand, we will prove the global existence of small data solutions for $p>p_{\text {Fuj }}(\mathbb{Q})$ in an exponential weighted energy space. On the other hand, a blow-up result for $1<p \leq p_{\text {Fuj }}(\mathbb{Q})$ under certain integral sign assumptions for the Cauchy data by using the test function method.
\end{abstract}

Keywords: damped wave equation, Heisenberg group, critical exponent, test function method, energy spaces with exponential weight.

2010 MSC: Primary: 35B33, 35L71, 35R03; Secondary: 35B44, 35B45, 43A80, 58J45.

\section{Introduction}

In this paper we study the global in time existence of small data solutions and the blow-up in finite time of solutions to the Cauchy problem

$$
\begin{cases}u_{t t}-\Delta_{\mathrm{H}} u+u_{t}=|u|^{p}, & t>0, \eta \in \mathbf{H}_{n}, \\ u(0, \eta)=u_{0}(\eta), & \eta \in \mathbf{H}_{n}, \\ u_{t}(0, \eta)=u_{1}(\eta), & \eta \in \mathbf{H}_{n},\end{cases}
$$

where $p>1$ and $\Delta_{\mathrm{H}}$ denotes the sub-Laplacian on $\mathbf{H}_{n}$ (see Subsection 1.1 for the definition of $\Delta_{\mathrm{H}}$ and for a short summary on the Heisenberg group).

In the Euclidean case, the critical exponent of Cauchy problem for the semilinear damped wave equations

$$
\begin{cases}u_{t t}-\Delta u+u_{t}=|u|^{p}, & t>0, x \in \mathbb{R}^{n}, \\ u(0, x)=u_{0}(x), & x \in \mathbb{R}^{n}, \\ u_{t}(0, x)=u_{1}(x), & x \in \mathbb{R}^{n},\end{cases}
$$

is the same as for the semilinear heat equations, that is, the so-called Fujita exponent

$$
p_{\text {Fuj }}(n) \doteq 1+\frac{2}{n} \text {. }
$$

Email addresses: vladimir.simeonov.gueorguiev@unipi.it (Vladimir Georgiev), alessandro.palmieri.math@gmail.com (Alessandro Palmieri) 
This fact has been proved by Todorova-Yordanov [21] for compactly supported data and by Ikehata-Tanizawa [12] in the not-compact case. In both works, the global existence result of small data solutions in the superFujita case is demonstrated in an exponentially weighted energy space. The crucial difference consists in the choice of the exponent function for the exponential weight. Furthermore, a fundamental tools in both these works are the decay estimates on $L^{2}\left(\mathbb{R}^{n}\right)$ - basis for the corresponding linear homogeneous Cauchy problem, that have been derived by Matsumura in the pioneering paper [13], by using phase space analysis.

This approach with exponential weighted energy spaces has been applied also the case of time-dependent coefficients: see [8] for the semilinear wave equation with effective damping and $[7,16]$ for the scale-invariant case, respectively.

Recently, it has been shown that the critical exponent for the semilinear heat equation on the Heisenberg group is the Fujita exponent $p_{\text {Fuj }}(\mathbb{Q})$, where $\mathbb{Q}$ is the homogeneous dimension of $\mathbf{H}_{n}$, and on more general stratified Lie groups (cf. [20, 10, 11]).

In this paper, we will show that $p_{\mathrm{Fuj}}(\mathbb{Q})$ is the critical exponent for the Cauchy problem $(1)$ as well. Concerning the existence of small data solutions which are globally defined in time for $p>p_{\text {Fuj }}(\mathbb{Q})$, we will adapt in a suitable way the approach of $[21,12]$ with exponential weights. In fact, the counterpart of Matsumura-type estimates for the Heisenberg group is considered in [17], where the group Fourier transform is employed in order to show decay estimates on $L^{2}\left(\mathbf{H}_{n}\right)$ - basis for the corresponding homogeneous linear Cauchy problem (cf. Proposition 6.1). On the other hand, the non-existence of global solutions when $1<p \leq p_{\text {Fuj }}(\mathbb{Q})$, under certain integral sign assumptions for the Cauchy data and regardless of the smallness of these, is obtained by using the so-called test function method (see [14] or, for example, [15, 6, 10, 11]).

Finally, we point out that in [19] a global existence result for small data solutions is proved in the more general frame of graded Lie groups for the semilinear damped wave equation with an additional mass term. For that model, no further lower bound for the exponent of the nonlinearity $p>1$ has to be required, due to the exponential decay rate in $L^{2}-L^{2}$ estimates for the corresponding linear homogeneous Cauchy problem (nevertheless, an upper bound for $p$ is required, although it is a technical assumption due to the application of an inequality of Gagliardo-Nirenberg type). We refer to [17] for further details on the differences that are produced by the absence of the mass term in the treatment of the corresponding linear problems.

\subsection{The Heisenberg group}

The Heisenberg group is the Lie group $\mathbf{H}_{n}=\mathbb{R}^{2 n+1}$ equipped with the multiplication rule

$$
(x, y, \tau) \circ\left(x^{\prime}, y^{\prime}, \tau^{\prime}\right)=\left(x+x^{\prime}, y+y^{\prime}, \tau+\tau^{\prime}+\frac{1}{2}\left(x \cdot y^{\prime}-x^{\prime} \cdot y\right)\right),
$$

where $\cdot$ denotes the standard scalar product in $\mathbb{R}^{n}$. A system of left-invariant vector fields that span the Lie algebra $\mathfrak{h}_{n}$ is given by

$$
X_{j} \doteq \partial_{x_{j}}-\frac{y_{j}}{2} \partial_{\tau}, Y_{j} \doteq \partial_{y_{j}}+\frac{x_{j}}{2} \partial_{\tau}, \partial_{\tau}
$$

where $1 \leq j \leq n$. This system satisfies the commutation relations

$$
\left[X_{j}, Y_{k}\right]=\delta_{j k} \partial_{\tau} \quad \text { for } 1 \leq j, k \leq n \text {. }
$$

Therefore, $\mathfrak{h}_{n}$ admits the stratification $\mathfrak{h}_{n}=V_{1} \oplus V_{2}$, where $V_{1} \doteq \operatorname{span}\left\{X_{j}, Y_{j}\right\}_{1 \leq j \leq n}$ and $V_{2} \doteq \operatorname{span}\left\{\partial_{\tau}\right\}$. Hence, $\mathbf{H}_{n}$ is a 2 step stratified Lie group, whose homogeneous dimension is $\mathbb{Q}=2 n+2$. The sub-Laplacian on $\mathbf{H}_{n}$ is defined as

$$
\Delta_{\mathrm{H}} \doteq \sum_{j=1}^{n}\left(X_{j}^{2}+Y_{j}^{2}\right)=\sum_{j=1}^{n}\left(\partial_{x_{j}}^{2}+\partial_{y_{j}}^{2}\right)+\frac{1}{4} \sum_{j=1}^{n}\left(x_{j}^{2}+y_{j}^{2}\right) \partial_{\tau}^{2}+\sum_{j=1}^{n}\left(x_{j} \partial_{y_{j} \tau}^{2}-y_{j} \partial_{x_{j} \tau}^{2}\right) .
$$

For a function $v: \mathbf{H}_{n} \rightarrow \mathbb{R}$, the horizontal gradient of $v$ is

$$
\nabla_{\mathrm{H}} v \doteq\left(X_{1} v, \cdots, X_{n} v, Y_{1} v, \cdots, Y_{n} v\right) \equiv \sum_{j=1}^{n}\left(\left(X_{j} v\right) X_{j}+\left(Y_{j} v\right) Y_{j}\right)
$$


where each fiber of the horizontal subbundle $\mathrm{HH}_{n}=\sqcup_{\eta \in \mathbf{H}_{n}} \mathrm{H}_{\eta} \mathbf{H}_{n}$ can be endowed with a scalar product $\langle\cdot, \cdot\rangle_{\eta}$ in such a way that $X_{1}(\eta), \cdots, X_{n}(\eta), Y_{1}(\eta), \cdots, Y_{n}(\eta)$ are orthonormal in $\left(\mathrm{H}_{\eta} \mathbf{H}_{n},\langle\cdot, \cdot\rangle_{\eta}\right)$ for any $\eta \in \mathbf{H}_{n}$. Therefore, if $X=\sum_{j=1}^{n}\left(\alpha_{j} X_{j}+\beta_{j} Y_{1}\right)+\gamma \partial_{\tau}$ is a vector field on $\mathbf{H}_{n}$ with $\alpha_{j}, \beta_{j}, \gamma \in \mathscr{C}^{1}\left(\mathbf{H}_{n}\right)$ for any $j=1, \cdots, n$, the divergence of $X$ is the function

$$
\operatorname{div} X \doteq \sum_{j=1}^{n}\left(X_{j} \alpha_{j}+Y_{j} \beta_{j}\right)+\partial_{\tau} \gamma
$$

In particular, the sub-Laplacian may be expressed also as $\Delta_{\mathrm{H}} v=\operatorname{div}\left(\nabla_{\mathrm{H}} v\right)$. For a function $v \in L^{2}\left(\mathbf{H}_{n}\right)$ we say that $X_{j} v, Y_{j} v \in L_{\mathrm{loc}}^{1}\left(\mathbf{H}_{n}\right)$ exist in the sense of distributions, if the integral relations

$$
\int_{\mathbf{H}_{\mathbf{n}}}\left(X_{j} v\right)(\eta) \phi(\eta) \mathrm{d} \eta=\int_{\mathbf{H}_{\mathbf{n}}} v(\eta)\left(X_{j}^{*} \phi\right)(\eta) \mathrm{d} \eta \quad \text { and } \quad \int_{\mathbf{H}_{\mathbf{n}}}\left(Y_{j} v\right)(\eta) \phi(\eta) \mathrm{d} \eta=\int_{\mathbf{H}_{\mathbf{n}}} v(\eta)\left(Y_{j}^{*} \phi\right)(\eta) \mathrm{d} \eta
$$

are fulfilled for any $\phi \in \mathscr{C}_{0}^{\infty}\left(\mathbf{H}_{n}\right)$, where $X_{j}^{*}=-X_{j}$ and $Y_{j}^{*}=-Y_{j}$ denote the formal adjoint operators of $X_{j}$ and $Y_{j}$, respectively. Therefore, in our framework, the Sobolev space $H^{1}\left(\mathbf{H}_{n}\right)$ is the set of all functions $v \in L^{2}\left(\mathbf{H}_{n}\right)$ such that $X_{j} v, Y_{j} v$ exist in the sense of distributions and $X_{j} v, Y_{j} v \in L^{2}\left(\mathbf{H}_{n}\right)$ for any $j=1, \cdots, n$, equipped with the norm

$$
\begin{aligned}
\|v\|_{H^{1}\left(\mathbf{H}_{n}\right)}^{2} & \doteq\|v\|_{L^{2}\left(\mathbf{H}_{n}\right)}^{2}+\left\|\nabla_{\mathrm{H}} v\right\|_{L^{2}\left(\mathbf{H}_{n}\right)}^{2} \\
& =\|v\|_{L^{2}\left(\mathbf{H}_{n}\right)}^{2}+\sum_{j=1}^{n}\left(\left\|X_{j} v\right\|_{L^{2}\left(\mathbf{H}_{n}\right)}^{2}+\left\|Y_{j} v\right\|_{L^{2}\left(\mathbf{H}_{n}\right)}^{2}\right) .
\end{aligned}
$$

\subsection{Notations}

In this paper, we write $f \lesssim g$, when there exists a constant $C>0$ such that $f \leq C g$. We write $f \approx g$ when $g \lesssim f \lesssim g$. Throughout the article we will denote by $\psi$ the function

$$
\psi(t, \eta) \doteq \frac{|x|^{2}+|y|^{2}+4|\tau|}{8(1+t)}
$$

for any $\eta=(x, y, \tau) \in \mathbf{H}_{n}$. Let $\sigma>0$ and $t \geq 0$. Similarly to the Euclidean case considered in [21] and [12], we define the Sobolev spaces $L^{2}$ and $H^{1}$ with exponential weight $\mathrm{e}^{\sigma \psi(t, \cdot)}$

$$
\begin{aligned}
L_{\sigma \psi(t, \cdot)}^{2}\left(\mathbf{H}_{n}\right) \doteq\left\{v \in L^{2}\left(\mathbf{H}_{n}\right):\left\|\mathrm{e}^{\sigma \psi(t, \cdot)} v\right\|_{L^{2}\left(\mathbf{H}_{n}\right)}<\infty\right\}, \\
H_{\sigma \psi(t, \cdot)}^{1}\left(\mathbf{H}_{n}\right) \doteq\left\{v \in H^{1}\left(\mathbf{H}_{n}\right):\left\|\mathrm{e}^{\sigma \psi(t, \cdot)} v\right\|_{L^{2}\left(\mathbf{H}_{n}\right)}+\left\|\mathrm{e}^{\sigma \psi(t, \cdot)} \nabla_{\mathrm{H}} v\right\|_{L^{2}\left(\mathbf{H}_{n}\right)}<\infty\right\},
\end{aligned}
$$

endowed with the norms

$$
\begin{aligned}
\|v\|_{L_{\sigma \psi(t, \cdot)}^{2}\left(\mathbf{H}_{n}\right)} & \doteq\left\|\mathrm{e}^{\sigma \psi(t, \cdot)} v\right\|_{L^{2}\left(\mathbf{H}_{n}\right)}, \\
\|v\|_{H_{\sigma \psi(t, \cdot)}^{1}\left(\mathbf{H}_{n}\right)} & \doteq\left\|\mathrm{e}^{\sigma \psi(t, \cdot)} v\right\|_{L^{2}\left(\mathbf{H}_{n}\right)}+\left\|\mathrm{e}^{\sigma \psi(t, \cdot)} \nabla_{\mathrm{H}} v\right\|_{L^{2}\left(\mathbf{H}_{n}\right)} .
\end{aligned}
$$

In the local and global existence results for (1) we will consider always the special case $\sigma=1$ for the function spaces to which solutions belong. Nonetheless, in order to deal with the estimates of the nonlinearity, it is necessary sometimes to consider the general case $\sigma>0$. Finally, we denote by $\mathscr{A}$ the space

$$
\mathscr{A}\left(\mathbf{H}_{n}\right) \doteq H_{\psi(0, \cdot)}^{1}\left(\mathbf{H}_{n}\right) \times L_{\psi(0, \cdot)}^{2}\left(\mathbf{H}_{n}\right)
$$

to which initial data will be required to belong to. 


\section{Main results}

Let us state the main theorems that will be proved in the present article.

Theorem 2.1. Let $n \geq 1$. Let us assume $p>1$ such that $p \leq p_{\mathrm{GN}}(\mathbb{Q}) \doteq \frac{\mathbb{Q}}{\mathbb{Q}-2}$. Then for each initial data $\left(u_{0}, u_{1}\right) \in \mathscr{A}\left(\mathbf{H}_{n}\right)$ there exists a maximal existence time $T_{\max } \in(0, \infty]$ such that the Cauchy problem (1) has a unique solution $u \in \mathscr{C}\left(\left[0, T_{\max }\right), H^{1}\left(\mathbf{H}_{n}\right)\right) \cap \mathscr{C}^{1}\left(\left[0, T_{\max }\right), L^{2}\left(\mathbf{H}_{n}\right)\right)$.

Moreover, for any $T \in\left(0, T_{\max }\right)$ it holds

$$
\sup _{t \in[0, T]}\left(\left\|\mathrm{e}^{\psi(t, \cdot)} v(t, \cdot)\right\|_{L^{2}\left(\mathbf{H}_{n}\right)}+\left\|\mathrm{e}^{\psi(t, \cdot)} \nabla_{\mathrm{H}} v(t, \cdot)\right\|_{L^{2}\left(\mathbf{H}_{n}\right)}+\left\|\mathrm{e}^{\psi(t, \cdot)} v_{t}(t, \cdot)\right\|_{L^{2}\left(\mathbf{H}_{n}\right)}\right)<\infty .
$$

Finally, if $T_{\max }<\infty$, then

$$
\limsup _{T \rightarrow T_{\max }^{-}}\left(\left\|\mathrm{e}^{\psi(t, \cdot)} v(t, \cdot)\right\|_{L^{2}\left(\mathbf{H}_{n}\right)}+\left\|\mathrm{e}^{\psi(t, \cdot)} \nabla_{\mathrm{H}} v(t, \cdot)\right\|_{L^{2}\left(\mathbf{H}_{n}\right)}+\left\|\mathrm{e}^{\psi(t, \cdot)} v_{t}(t, \cdot)\right\|_{L^{2}\left(\mathbf{H}_{n}\right)}\right)=\infty .
$$

The previous local existence result is a preparatory result to the next global existence theorem, whose proof is based on a contradiction argument that requires the existence of local in time solutions for (1).

Theorem 2.2. Let $n \geq 1$. Let us consider $1<p \leq p_{\mathrm{GN}}(\mathbb{Q})$ such that $p>p_{\text {Fuj }}(\mathbb{Q})$. Then, there exists $\varepsilon_{0}>0$ such that for any initial data

$$
\left(u_{0}, u_{1}\right) \in \mathscr{A}\left(\mathbf{H}_{n}\right) \quad \text { satisfying } \quad\left\|\left(u_{0}, u_{1}\right)\right\|_{\mathscr{A}\left(\mathbf{H}_{n}\right)} \leq \varepsilon_{0}
$$

there is a unique solution $u \in \mathscr{C}\left([0, \infty), H_{\psi(t, \cdot)}^{1}\left(\mathbf{H}_{n}\right)\right) \cap \mathscr{C}^{1}\left([0, \infty), L_{\psi(t, \cdot)}^{2}\left(\mathbf{H}_{n}\right)\right)$ to the Cauchy problem (1). Moreover, u satisfies the following estimates

$$
\begin{aligned}
& \|u(t, \cdot)\|_{L^{2}\left(\mathbf{H}_{n}\right)} \lesssim(1+t)^{-\frac{\widehat{Q}}{2}}\left\|\left(u_{0}, u_{1}\right)\right\|_{\mathscr{A}\left(\mathbf{H}_{n}\right)}, \\
& \left\|\nabla_{\mathrm{H}} u(t, \cdot)\right\|_{L^{2}\left(\mathbf{H}_{n}\right)} \lesssim(1+t)^{-\frac{0}{4}-\frac{1}{2}}\left\|\left(u_{0}, u_{1}\right)\right\|_{\mathcal{A}\left(\mathbf{H}_{n}\right)}, \\
& \left\|u_{t}(t, \cdot)\right\|_{L^{2}\left(\mathbf{H}_{n}\right)} \lesssim(1+t)^{-\frac{\Omega}{4}-1}\left\|\left(u_{0}, u_{1}\right)\right\|_{\mathcal{S}\left(\mathbf{H}_{n}\right)}, \\
& \left\|\mathrm{e}^{\psi(t, \cdot)} \nabla_{\mathrm{H}} u(t, \cdot)\right\|_{L^{2}\left(\mathbf{H}_{n}\right)} \lesssim\left\|\left(u_{0}, u_{1}\right)\right\|_{\mathcal{A}\left(\mathbf{H}_{n}\right)}, \\
& \left\|\mathrm{e}^{\psi(t, \cdot)} u_{t}(t, \cdot)\right\|_{L^{2}\left(\mathbf{H}_{n}\right)} \lesssim\left\|\left(u_{0}, u_{1}\right)\right\|_{\mathscr{S}\left(\mathbf{H}_{n}\right)}
\end{aligned}
$$

for any $t \geq 0$.

Remark 1. Let us point out that the requirement $\left(u_{0}, u_{1}\right) \in \mathscr{A}\left(\mathbf{H}_{n}\right)$ in Theorem 2.2 is stronger than the assumption $\left(u_{0}, u_{1}\right) \in\left(H^{1}\left(\mathbf{H}_{n}\right) \cap L^{1}\left(\mathbf{H}_{n}\right)\right) \times\left(L^{2}\left(\mathbf{H}_{n}\right) \cap L^{1}\left(\mathbf{H}_{n}\right)\right)$. Indeed, the embedding

$$
L_{\sigma \psi(t, .)}^{2}\left(\mathbf{H}_{n}\right) \hookrightarrow L^{1}\left(\mathbf{H}_{n}\right) \cap L^{2}\left(\mathbf{H}_{n}\right)
$$

holds for any $\sigma>0$ and $t \geq 0$. By using Cauchy-Schwarz inequality and the nonnegativity of $\psi$, it results

$$
\begin{aligned}
\|v\|_{L^{1}\left(\mathbf{H}_{n}\right)} & \lesssim(1+t)^{\frac{9}{4}}\left\|\mathrm{e}^{\sigma \psi(t, \cdot)} v\right\|_{L^{2}\left(\mathbf{H}_{n}\right)}, \\
\|v\|_{L^{2}\left(\mathbf{H}_{n}\right)} & \lesssim\left\|\mathrm{e}^{\sigma \psi(t, \cdot)} v\right\|_{L^{2}\left(\mathbf{H}_{n}\right)} .
\end{aligned}
$$

In order to prove (7), we employed the value of the integral of Gaussian-type

$$
\begin{aligned}
\int_{\mathbf{H}_{n}} \mathrm{e}^{-2 \sigma \psi(t, \eta)} \mathrm{d} \eta & =\int_{\mathbb{R}^{n}} \mathrm{e}^{-\frac{\sigma|x|^{2}}{4(1+t)}} \mathrm{d} x \int_{\mathbb{R}^{n}} \mathrm{e}^{-\frac{\sigma|y|^{2}}{4(1+t)}} \mathrm{d} y \int_{\mathbb{R}} \mathrm{e}^{-\frac{\sigma|\tau|}{4(1+t)}} \mathrm{d} \tau \\
& =2^{Q+1} \pi^{\frac{\Theta}{2}-1} \sigma^{-\frac{\Theta}{2}}(1+t)^{\frac{\Theta}{2}}
\end{aligned}
$$

Furthermore, by Hölder's interpolation inequality we have also the embedding of $L_{\sigma \psi(t, \cdot)}^{2}\left(\mathbf{H}_{n}\right)$ in each $L^{r}\left(\mathbf{H}_{n}\right)$ for any $r \in[1,2]$, where the embedding constant depends on $t$, clearly. 
Theorem 2.3. Let $n \geq 1$. Let $u_{0}, u_{1} \in L^{1}\left(\mathbf{H}_{n}\right)$ such that

$$
\liminf _{R \rightarrow \infty} \int_{\mathscr{D}_{R}}\left(u_{0}(\eta)+u_{1}(\eta)\right) \mathrm{d} \eta>0
$$

where $\mathscr{D}_{R} \doteq B_{n}(R) \times B_{n}(R) \times\left[-R^{2}, R^{2}\right]$. Let us assume that $u \in L_{\text {loc }}^{p}\left([0, T) \times \mathbb{R}^{n}\right)$ is a solution to (1), with life-span $T>0$. If $1<p \leq p_{\mathrm{Fuj}}(Q)$, then $T<\infty$, that is, the solutions $u$ blows up in finite time.

The next sections are organized as follows: in Section 3 we explain the strategy for the proofs of Theorems 2.1 and 2.2 and we derive some important estimates by using some remarkable properties of the function $\psi$; in Sections 4 and 6 we derive a weighted version of the Gagliardo-Nirenberg inequality on $\mathbf{H}_{n}$ and we recall $L^{2}\left(\mathbf{H}_{n}\right)-L^{2}\left(\mathbf{H}_{n}\right)$ estimates (with possible additional $L^{1}\left(\mathbf{H}_{n}\right)$ regularity) for the solution of (10), respectively; then, we prove Theorems 2.1 and 2.2 in Section 5 and in Section 7, respectively; finally, we prove the blow-up result in Section 8.

\section{Overview on our approach}

We apply Duhamel's principle in order to write the solution to (1). Because the linear equation related to the semi-linear equation in (1) is invariant by time translations, we need to derive decay estimates for linear Cauchy problem

$$
\begin{cases}u_{t t}-\Delta_{\mathrm{H}} u+u_{t}=0, & t>0, \eta \in \mathbf{H}_{n}, \\ u(0, \eta)=u_{0}(\eta), & \eta \in \mathbf{H}_{n}, \\ u_{t}(0, \eta)=u_{1}(\eta), & \eta \in \mathbf{H}_{n} .\end{cases}
$$

Let us fix now some notations for the linear Cauchy problem (10). We denote by $E_{0}(t, \eta), E_{1}(t, \eta)$ the fundamental solutions to the Cauchy problem (10), i.e., the distributional solutions with data $\left(u_{0}, u_{1}\right)=$ $\left(\delta_{0}, 0\right)$ and $\left(u_{0}, u_{1}\right)=\left(0, \delta_{0}\right)$, respectively, where $\delta_{0}$ is the Dirac distribution in the $\eta$ variable. Also, if we denote by $*_{(\eta)}$ the group convolution with respect to the $\eta$ variable, we may represent the solution to the Cauchy problem (10) as

$$
u(t, \eta)=u_{0}(\eta) *_{(\eta)} E_{0}(t, \eta)+u_{1}(\eta) *_{(\eta)} E_{1}(t, \eta)
$$

According to Duhamel's principle adapted to the case of Lie groups, we get

$$
u(t, \eta)=\int_{0}^{t} F(s, \eta) *(\eta) E_{1}(t-s, \eta) \mathrm{d} s
$$

as mild solution to the inhomogeneous Cauchy problem

$$
\begin{cases}u_{t t}-\Delta_{\mathrm{H}} u+u_{t}=F(t, \eta), & t>0, \eta \in \mathbf{H}_{n}, \\ u(0, \eta)=0, & \eta \in \mathbf{H}_{n}, \\ u_{t}(0, \eta)=0, & \eta \in \mathbf{H}_{n} .\end{cases}
$$

In particular, we used the fact that the identity $L\left(v *_{(\eta)} E_{1}\right)=v *_{(\eta)} L\left(E_{1}\right)$ holds for any left invariant differential operator $L$ on $\mathbf{H}_{n}$.

Therefore, we consider as mild solutions to $(1)$ on $(0, T) \times \mathbf{H}_{n}$ any fixed point of the nonlinear integral operator $N$ defined as follows:

$$
u \in X(T) \rightarrow N u(t, \eta) \doteq u_{0}(\eta) *_{(\eta)} E_{0}(t, \eta)+u_{1}(\eta) *_{(\eta)} E_{1}(t, \eta)+\int_{0}^{t}|u(s, \eta)|^{p} *_{(\eta)} E_{1}(t-s, \eta) \mathrm{d} s
$$

for a suitably chosen space $X(T)$ (here $T$ denotes the lifespan of the solution). 
In particular, in Theorem 2.2 the global in time solutions we are interested in are solution in $X(T)$ to the integral equation

$$
u(t, \eta)=u_{0}(\eta) *_{(\eta)} E_{0}(t, \eta)+u_{1}(\eta) *_{(\eta)} E_{1}(t, \eta)+\int_{0}^{t}|u(s, \eta)|^{p} *_{(\eta)} E_{1}(t-s, \eta) \mathrm{d} s
$$

which can be extended for all positive times.

Also, one difficulty in the proof of the local existence result for large data and of the global existence result for small data, respectively, consists in the choice of the space $X(T)$. In this paper, we restrict our consideration to the weighted energy space

$$
X(T)=\mathscr{C}\left([0, T], H_{\psi(t, \cdot)}^{1}\left(\mathbf{H}_{n}\right)\right) \cap \mathscr{C}^{1}\left([0, T], L_{\psi(t, \cdot)}^{2}\left(\mathbf{H}_{n}\right)\right),
$$

both in Theorem 2.1 and in Theorem 2.2. As we will see, the crucial difference lies in the choice of the norm for $X(T)$ (cf. Section 5 and Section 7).

We analyze now some properties of the function $\psi$, defined in (4), that will be useful in the proof of our main results. Straightforward computations lead to

$$
\psi_{t}(t, \eta)=-\frac{|x|^{2}+|y|^{2}+4|\tau|}{8(1+t)^{2}}, \quad X_{j} \psi(t, \eta)=\frac{x_{j}-\operatorname{sign}(\tau) y_{j}}{4(1+t)}, \quad Y_{j} \psi(t, \eta)=\frac{y_{j}+\operatorname{sign}(\tau) x_{j}}{4(1+t)}
$$

for any $j=1, \cdots, n$. Let us point out explicitly that in the following we consider weak derivatives, so, the previous expressions for $X_{j} \psi$ and $Y_{j} \psi$ are in the sense of distributions. Consequently, the following inequalities are satisfied

$$
\begin{aligned}
\left|\nabla_{\mathrm{H}} \psi(t, \eta)\right|^{2}+\psi_{t}(t, \eta) & =-\frac{|\tau|}{2(1+t)^{2}} \leq 0, \\
\Delta_{\mathrm{H}} \psi(t, \eta) & =\frac{n}{2(1+t)}+\frac{|x|^{2}+|y|^{2}}{4(1+t)} \delta_{0}(\tau)
\end{aligned}
$$

for any $t \geq 0$ and any $\eta \in \mathbf{H}_{n}$, where $\delta_{0}(\tau)$ denotes the Dirac delta in 0 with respect to the $\tau$ variable. We derive now some fundamental relations that will play a crucial role in the next sections. The first one is the identity

$$
\begin{aligned}
\mathrm{e}^{2 \psi} u_{t}\left(u_{t t}-\Delta_{\mathrm{H}} u+u_{t}\right)= & \frac{\partial}{\partial t}\left(\frac{\mathrm{e}^{2 \psi}}{2}\left(\left|u_{t}\right|^{2}+\left|\nabla_{\mathrm{H}} u\right|^{2}\right)\right)-\operatorname{div}\left(\mathrm{e}^{2 \psi} u_{t} \nabla_{\mathrm{H}} u\right)+\frac{\mathrm{e}^{2 \psi}}{\psi_{t}} u_{t}^{2}\left(\left|\nabla_{\mathrm{H}} \psi\right|^{2}+\psi_{t}\right) \\
& -\psi_{t} \mathrm{e}^{2 \psi} u_{t}^{2}-\frac{\mathrm{e}^{2 \psi}}{\psi_{t}}\left|u_{t} \nabla_{\mathrm{H}} \psi-\psi_{t} \nabla_{\mathrm{H}} u\right|^{2},
\end{aligned}
$$

where $\left|\nabla_{\mathrm{H}} v\right|^{2}=\sum_{j=1}^{n}\left(\left|X_{j} v\right|^{2}+\left|Y_{j} v\right|^{2}\right)$ is the Euclidean norm of $\nabla_{\mathrm{H}} v$ provided that we use the identification $\nabla_{\mathrm{H}} v \simeq\left(X_{1} u, \cdots, X_{n} u, Y_{1} u, \cdots, Y_{n} u\right): \mathbf{H}_{n} \rightarrow \mathbb{R}^{2 n}$ or, equivalently, we consider on each fiber of the horizontal subbundle $\mathrm{H}_{\eta} \mathbf{H}_{n}$ the norm induced by the scalar product $\langle\cdot, \cdot\rangle_{\eta}$. Let us verify the validity of (15). Using the fact that the sub-Laplacian can be expressed as the divergence of the horizontal gradient and the identity

$$
\operatorname{div}(\alpha X)=\alpha \operatorname{div} X+X(\alpha)
$$

for any $\alpha \in \mathscr{C}^{1}\left(\mathbf{H}_{n}\right)$ and any horizontal vector field $X$ on $\mathbf{H}_{n}$, we get

$$
\begin{aligned}
\mathrm{e}^{\psi} u_{t} \Delta_{\mathrm{H}} u & =\mathrm{e}^{\psi} u_{t} \operatorname{div}\left(\nabla_{\mathrm{H}} u\right)=\operatorname{div}\left(\mathrm{e}^{2 \psi} u_{t} \nabla_{\mathrm{H}} u\right)-\left(\nabla_{\mathrm{H}} u\right)\left(\mathrm{e}^{2 \psi} u_{t}\right) \\
& =\operatorname{div}\left(\mathrm{e}^{2 \psi} u_{t} \nabla_{\mathrm{H}} u\right)-\left(\sum_{j=1}^{n} X_{j}(u) X_{j}+Y_{j}(u) Y_{j}\right)\left(\mathrm{e}^{2 \psi} u_{t}\right) \\
& =\operatorname{div}\left(\mathrm{e}^{2 \psi} u_{t} \nabla_{\mathrm{H}} u\right)-\sum_{j=1}^{n} X_{j}(u)\left(\mathrm{e}^{2 \psi} X_{j}\left(u_{t}\right)+2 \mathrm{e}^{2 \psi} u_{t} X_{j}(\psi)\right)-\sum_{j=1}^{n} Y_{j}(u)\left(\mathrm{e}^{2 \psi} Y_{j}\left(u_{t}\right)+2 \mathrm{e}^{2 \psi} u_{t} Y_{j}(\psi)\right) .
\end{aligned}
$$


Since

$$
\begin{aligned}
\sum_{j=1}^{n} X_{j}(u) X_{j}\left(u_{t}\right) \mathrm{e}^{2 \psi} & =\sum_{j=1}^{n} \frac{\mathrm{e}^{2 \psi}}{2} \frac{\partial}{\partial t}\left|X_{j}(u)\right|^{2} \\
& =\sum_{j=1}^{n}\left(\frac{\partial}{\partial t}\left(\frac{\mathrm{e}^{2 \psi}}{2}\left|X_{j}(u)\right|^{2}\right)-\psi_{t} \mathrm{e}^{2 \psi}\left|X_{j}(u)\right|^{2}\right)
\end{aligned}
$$

and, analogously,

$$
\sum_{j=1}^{n} Y_{j}(u) Y_{j}\left(u_{t}\right) \mathrm{e}^{2 \psi}=\sum_{j=1}^{n}\left(\frac{\partial}{\partial t}\left(\frac{\mathrm{e}^{2 \psi}}{2}\left|Y_{j}(u)\right|^{2}\right)-\psi_{t} \mathrm{e}^{2 \psi}\left|Y_{j}(u)\right|^{2}\right)
$$

it follows

$$
\sum_{j=1}^{n}\left(X_{j}(u) X_{j}\left(u_{t}\right) \mathrm{e}^{2 \psi}+Y_{j}(u) Y_{j}\left(u_{t}\right) \mathrm{e}^{2 \psi}\right)=\frac{\partial}{\partial t}\left(\frac{\mathrm{e}^{2 \psi}}{2}\left|\nabla_{\mathrm{H}} u\right|^{2}\right)-\psi_{t} \mathrm{e}^{2 \psi}\left|\nabla_{\mathrm{H}} u\right|^{2} .
$$

On the other hand, using the $(0,2)$ symmetric tensor $\langle\cdot, \cdot\rangle$ on $\mathbf{H}_{n}$, whose restriction to each fiber $\mathrm{H}_{\eta} \mathbf{H}_{n}$ of the horizontal subbundle is the scalar product $\langle\cdot, \cdot\rangle_{\eta}$ with orthonormal basis given by the canonical generators of the horizontal layer (cf. Subsection 1.1), we have

$$
\left\langle u_{t} \nabla_{\mathrm{H}} \psi, \psi_{t} \nabla_{\mathrm{H}} u\right\rangle=\sum_{j=1}^{n}\left(u_{t} \psi_{t} X_{j}(\psi) X_{j}(u)+u_{t} \psi_{t} Y_{j}(\psi) Y_{j}(u)\right) .
$$

Consequently,

$$
\begin{aligned}
2 \sum_{j=1}^{n} \mathrm{e}^{2 \psi} u_{t} X_{j}(\psi) X_{j}(u)+\mathrm{e}^{2 \psi} u_{t} Y_{j}(\psi) Y_{j}(u) & =2 \frac{\mathrm{e}^{2 \psi}}{\psi_{t}}\left\langle u_{t} \nabla_{\mathrm{H}} \psi, \psi_{t} \nabla_{\mathrm{H}} u\right\rangle \\
& =\frac{\mathrm{e}^{2 \psi}}{\psi_{t}}\left(u_{t}^{2}\left|\nabla_{\mathrm{H}} \psi\right|^{2}+\psi_{t}^{2}\left|\nabla_{\mathrm{H}} u\right|^{2}-\left|u_{t} \nabla_{\mathrm{H}} \psi-\psi_{t} \nabla_{\mathrm{H}} u\right|^{2}\right) .
\end{aligned}
$$

Combining (16), (17) and (18), we get

$$
\mathrm{e}^{\psi} u_{t} \Delta_{\mathrm{H}} u=\operatorname{div}\left(\mathrm{e}^{2 \psi} u_{t} \nabla_{\mathrm{H}} u\right)-\frac{\partial}{\partial t}\left(\frac{\mathrm{e}^{2 \psi}}{2}\left|\nabla_{\mathrm{H}} u\right|^{2}\right)+\frac{\mathrm{e}^{2 \psi}}{\psi_{t}}\left(\left|u_{t} \nabla_{\mathrm{H}} \psi-\psi_{t} \nabla_{\mathrm{H}} u\right|^{2}-u_{t}^{2}\left|\nabla_{\mathrm{H}} \psi\right|^{2}\right) .
$$

Furthermore,

$$
\mathrm{e}^{\psi} u_{t} u_{t t}=\frac{\mathrm{e}^{2 \psi}}{2} \partial_{t}\left|u_{t}\right|^{2}=\frac{\partial}{\partial t}\left(\frac{\mathrm{e}^{2 \psi}}{2}\left|u_{t}\right|^{2}\right)-\psi_{t} \mathrm{e}^{2 \psi} u_{t}^{2}
$$

By (19) and (20) we find immediately (15).

The second fundamental relation is the upcoming inequality, that is obtained by plugging the nonlinear term on the left hand side of (15). If $u$ is a solution of the equation (1), since

$$
\mathrm{e}^{2 \psi} u_{t}|u|^{p}=\mathrm{e}^{2 \psi} \partial_{t}\left(\frac{|u|^{p} u}{p+1}\right)=\frac{\partial}{\partial t}\left(\mathrm{e}^{2 \psi} \frac{|u|^{p} u}{p+1}\right)-2 \psi_{t} \mathrm{e}^{2 \psi} \frac{|u|^{p} u}{p+1},
$$

then, from (13) we get immediately

$$
\begin{aligned}
& \frac{\partial}{\partial t}\left(\frac{\mathrm{e}^{2 \psi}}{2}\left(\left|u_{t}\right|^{2}+\left|\nabla_{\mathrm{H}} u\right|^{2}\right)-\mathrm{e}^{2 \psi} \frac{|u|^{p} u}{p+1}\right) \\
& \quad=\operatorname{div}\left(\mathrm{e}^{2 \psi} u_{t} \nabla_{\mathrm{H}} u\right)-\frac{\mathrm{e}^{2 \psi}}{\psi_{t}} u_{t}^{2}\left(\left|\nabla_{\mathrm{H}} \psi\right|^{2}+\psi_{t}\right)+\psi_{t} \mathrm{e}^{2 \psi} u_{t}^{2}+\frac{\mathrm{e}^{2 \psi}}{\psi_{t}}\left|u_{t} \nabla_{\mathrm{H}} \psi-\psi_{t} \nabla_{\mathrm{H}} u\right|^{2}-2 \psi_{t} \mathrm{e}^{2 \psi} \frac{|u|^{p} u}{p+1} \\
& \quad \leq \operatorname{div}\left(\mathrm{e}^{2 \psi} u_{t} \nabla_{\mathrm{H}} u\right)-2 \psi_{t} \mathrm{e}^{2 \psi} \frac{|u|^{p} u}{p+1},
\end{aligned}
$$


where in the last inequality we used (13) and $\psi_{t} \leq 0$. We stress that in Sections 5 and 7 an important role in the derivation of weighted energy estimates will be played by (15) and (21).

\section{Gagliardo-Nirenberg type inequalities}

In the proof of Theorems 2.1 and 2.2, we make use of the following inequalities of Gagliardo-Nirenberg type. We begin with the Gagliardo-Nirenberg inequality in $\mathbf{H}_{n}$ (cf. $[5,19]$ ).

Lemma 4.1. Let $n \geq 1$. Let us consider $2 \leq q \leq 2+\frac{2}{n}=\frac{2 Q}{Q-2}$. Then, the following Gagliardo-Nirenberg inequality holds

$$
\|v\|_{L^{q}\left(\mathbf{H}_{n}\right)} \leq C\left\|\nabla_{\mathrm{H}} v\right\|_{L^{2}\left(\mathbf{H}_{n}\right)}^{\theta(q)}\|v\|_{L^{2}\left(\mathbf{H}_{n}\right)}^{1-\theta(q)}
$$

for any $v \in H^{1}\left(\mathbf{H}_{n}\right)$, where where $C$ is a nonnegative constant and $\theta(q) \in[0,1]$ is defined by

$$
\theta(q) \doteq \mathbb{Q}\left(\frac{1}{2}-\frac{1}{q}\right) .
$$

Lemma 4.2. Let $\sigma>0, t \geq 0$. Then, the following estimate

$$
2^{-1} \sigma n(1+t)^{-1}\left\|\mathrm{e}^{\sigma \psi(t, \cdot)} v\right\|_{L^{2}\left(\mathbf{H}_{n}\right)}^{2}+\left\|\nabla_{\mathrm{H}}\left(\mathrm{e}^{\sigma \psi(t, \cdot)} v\right)\right\|_{L^{2}\left(\mathbf{H}_{n}\right)}^{2} \leq\left\|\mathrm{e}^{\sigma \psi(t, \cdot)} \nabla_{\mathrm{H}} v\right\|_{L^{2}\left(\mathbf{H}_{n}\right)}^{2}
$$

holds for any $v \in H_{\sigma \psi(t, \cdot)}^{1}\left(\mathbf{H}_{n}\right)$.

Proof. Let us set $f=\mathrm{e}^{\sigma \psi} v$. Then, straightforward computations lead to

$$
\mathrm{e}^{\sigma \psi} \nabla_{\mathrm{H}} v=\nabla_{\mathrm{H}} f-\sigma f \nabla_{\mathrm{H}} \psi \text {. }
$$

Hence,

$$
\begin{aligned}
\left\|\mathrm{e}^{\sigma \psi(t, \cdot)} \nabla_{\mathrm{H}} v\right\|_{L^{2}\left(\mathbf{H}_{n}\right)}^{2} & =\left\|\nabla_{\mathrm{H}} f(t, \cdot)\right\|_{L^{2}\left(\mathbf{H}_{n}\right)}^{2}+\sigma^{2}\left\|\left(f \nabla_{\mathrm{H}} \psi\right)(t, \cdot)\right\|_{L^{2}\left(\mathbf{H}_{n}\right)}^{2}-2 \sigma\left(\nabla_{\mathrm{H}} f(t, \cdot),\left(f \nabla_{\mathrm{H}} \psi\right)(t, \cdot)\right)_{L^{2}\left(\mathbf{H}_{n}\right)} \\
& \geq\left\|\nabla_{\mathrm{H}} f(t, \cdot)\right\|_{L^{2}\left(\mathbf{H}_{n}\right)}^{2}-2 \sigma\left(\nabla_{\mathrm{H}} f(t, \cdot),\left(f \nabla_{\mathrm{H}} \psi\right)(t, \cdot)\right)_{L^{2}\left(\mathbf{H}_{n}\right)} .
\end{aligned}
$$

Integrating by parts, we have

Note that we may integrate by parts

$$
\int_{\mathbf{H}_{n}} X_{j} g(\eta) \cdot h(\eta) \mathrm{d} \eta=-\int_{\mathbf{H}_{n}} g(\eta) \cdot X_{j} h(\eta) \mathrm{d} \eta, \quad \int_{\mathbf{H}_{n}} Y_{j} g(\eta) \cdot h(\eta) \mathrm{d} \eta=-\int_{\mathbf{H}_{n}} g(\eta) \cdot Y_{j} h(\eta) \mathrm{d} \eta
$$

for any $g, h \in \mathscr{C}_{0}^{1}\left(\mathbf{H}_{n}\right)$ and for any $j=1, \cdots, n$. So, using a partition of the unity we may remove the compact support assumption while a density argument provides the result for weak derivatives.

$$
\begin{aligned}
\left(\nabla_{\mathrm{H}} f(t, \cdot),\right. & \left.\left(f \nabla_{\mathrm{H}} \psi\right)(t, \cdot)\right)_{L^{2}\left(\mathbf{H}_{n}\right)} \\
& =\sum_{j=1}^{n} \int_{\mathbf{H}_{n}}\left(f X_{j} f X_{j} \psi+f Y_{j} f Y_{j} \psi\right)(t, \eta) \mathrm{d} \eta=\frac{1}{2} \sum_{j=1}^{n} \int_{\mathbf{H}_{n}}\left(X_{j}|f|^{2} X_{j} \psi+Y_{j}|f|^{2} Y_{j} \psi\right)(t, \eta) \mathrm{d} \eta \\
& =-\frac{1}{2} \sum_{j=1}^{n} \int_{\mathbf{H}_{n}}\left(|f|^{2} X_{j}^{2} \psi+|f|^{2} Y_{j}^{2} \psi\right)(t, \eta) \mathrm{d} \eta=-\frac{1}{2} \int_{\mathbf{H}_{n}}\left(|f|^{2} \Delta_{\mathbf{H}} \psi\right)(t, \eta) \mathrm{d} \eta \\
& \leq-\frac{n}{4(1+t)}\|f(t, \cdot)\|_{L^{2}\left(\mathbf{H}_{n}\right)}^{2}
\end{aligned}
$$

where in the last step we used (14). Note that we may consider the trace of the function $|f|^{2}$ on the hypersurface with equation $\tau=0$, since the existence of trace operators is known in the literature for the Heisenberg group (cf. $[18,3,1,2]$ ). Consequently, combining (23) and (24), we get the desired estimate. 
Lemma 4.3. Let $n \geq 1, \sigma \in(0,1]$ and $t \geq 0$. Let us consider $2 \leq q \leq 2+\frac{2}{n}=\frac{2 Q}{Q-2}$. Then, the following weighted Gagliardo-Nirenberg inequality

$$
\left\|\mathrm{e}^{\sigma \psi(t, \cdot)} v\right\|_{L^{q}\left(\mathbf{H}_{n}\right)} \leq C(1+t)^{(1-\theta(q)) / 2}\left\|\nabla_{\mathrm{H}} v\right\|_{L^{2}\left(\mathbf{H}_{n}\right)}^{1-\sigma}\left\|\mathrm{e}^{\psi(t, \cdot)} \nabla_{\mathrm{H}} v\right\|_{L^{2}\left(\mathbf{H}_{n}\right)}^{\sigma},
$$

holds for any $v \in H_{\psi(t, \cdot)}^{1}\left(\mathbf{H}_{n}\right)$, where $C$ is a nonnegative constant and $\theta(q)$ is defined by (22).

Proof. Let us prove first that $v \in H_{\psi(t, \cdot)}^{1}\left(\mathbf{H}_{n}\right)$ implies $v \in H_{\sigma \psi(t, \cdot)}^{1}\left(\mathbf{H}_{n}\right)$ for any $\sigma \in(0,1]$. By Hölder's inequality we find

$$
\begin{aligned}
\left\|\mathrm{e}^{\sigma \psi(t, \cdot)} \nabla_{\mathrm{H}} v\right\|_{L^{2}\left(\mathbf{H}_{n}\right)}^{2} & =\int_{\mathbf{H}_{n}} \mathrm{e}^{2 \sigma \psi(t, \eta)}\left|\nabla_{\mathrm{H}} v(\eta)\right|^{2 \sigma}\left|\nabla_{\mathrm{H}} v(\eta)\right|^{2(1-\sigma)} \mathrm{d} \eta \\
& \leq\left\|\mathrm{e}^{2 \sigma \psi(t, \cdot)}\left|\nabla_{\mathrm{H}} v\right|^{2 \sigma}\right\|_{L^{\frac{1}{\sigma}}\left(\mathbf{H}_{n}\right)}\left\|\left|\nabla_{\mathrm{H}} v\right|^{2(1-\sigma)}\right\|_{L^{\frac{1}{1-\sigma}}\left(\mathbf{H}_{n}\right)} \\
& =\left\|\mathrm{e}^{\psi(t, \cdot)} \nabla_{\mathrm{H}} v\right\|_{L^{2}\left(\mathbf{H}_{n}\right)}^{2 \sigma}\left\|\nabla_{\mathrm{H}} v\right\|_{L^{2}\left(\mathbf{H}_{n}\right)}^{2(1-\sigma)} .
\end{aligned}
$$

In a similar way, it results

$$
\begin{aligned}
\left\|\mathrm{e}^{\sigma \psi(t, \cdot)} v\right\|_{L^{2}\left(\mathbf{H}_{n}\right)}^{2} & =\int_{\mathbf{H}_{n}} \mathrm{e}^{2 \sigma \psi(t, \eta)}|v(\eta)|^{2 \sigma}|v(\eta)|^{2(1-\sigma)} \mathrm{d} \eta \\
& \leq\left\|\mathrm{e}^{2 \sigma \psi(t, \cdot)}|v|^{2 \sigma}\right\|_{L^{\frac{1}{\sigma}}\left(\mathbf{H}_{n}\right)}\left\||v|^{2(1-\sigma)}\right\|_{L^{1-\sigma}\left(\mathbf{H}_{n}\right)} \\
& =\left\|\mathrm{e}^{\psi(t, \cdot)} v\right\|_{L^{2}\left(\mathbf{H}_{n}\right)}^{2 \sigma}\|v\|_{L^{2}\left(\mathbf{H}_{n}\right)}^{2(1-\sigma)} .
\end{aligned}
$$

So, we have that $f=\mathrm{e}^{\sigma \psi} v$ satisfies $f(t, \cdot) \in H^{1}\left(\mathbf{H}_{n}\right)$ and by Lemma 4.2

$$
\begin{aligned}
\|f(t, \cdot)\|_{L^{2}\left(\mathbf{H}_{n}\right)} & \lesssim(1+t)^{1 / 2}\left\|\mathrm{e}^{\sigma \psi(t, \cdot)} \nabla_{\mathrm{H}} v\right\|_{L^{2}\left(\mathbf{H}_{n}\right)}, \\
\left\|\nabla_{\mathrm{H}} f(t, \cdot)\right\|_{L^{2}\left(\mathbf{H}_{n}\right)} & \leq\left\|\mathrm{e}^{\sigma \psi(t, \cdot)} \nabla_{\mathrm{H}} v\right\|_{L^{2}\left(\mathbf{H}_{n}\right)}
\end{aligned}
$$

for any $t \geq 0$. Applying the Gagliardo-Nirenberg inequality to $f(t, \cdot)$ from Lemma 4.1, we have

$$
\|f(t, \cdot)\|_{L^{q}\left(\mathbf{H}_{n}\right)} \lesssim\|f(t, \cdot)\|_{L^{2}\left(\mathbf{H}_{n}\right)}^{1-\theta(q)}\left\|\nabla_{\mathrm{H}} f(t, \cdot)\right\|_{L^{2}\left(\mathbf{H}_{n}\right)}^{\theta(q)},
$$

where $\theta(q)=\mathbb{Q}\left(\frac{1}{2}-\frac{1}{q}\right)$. Also, combining (27) and (28) with the last interpolative inequality, we obtain

$$
\begin{aligned}
\|f(t, \cdot)\|_{L^{q}\left(\mathbf{H}_{n}\right)} & \lesssim(1+t)^{(1-\theta(q)) / 2}\left\|\mathrm{e}^{\sigma \psi(t, \cdot)} \nabla_{\mathrm{H}} v\right\|_{L^{2}\left(\mathbf{H}_{n}\right)} \\
& \leq(1+t)^{(1-\theta(q)) / 2}\left\|\mathrm{e}^{\psi(t, \cdot)} \nabla_{\mathrm{H}} v\right\|_{L^{2}\left(\mathbf{H}_{n}\right)}^{\sigma}\left\|\nabla_{\mathrm{H}} v\right\|_{L^{2}\left(\mathbf{H}_{n}\right)}^{1-\sigma},
\end{aligned}
$$

where in the last step we applied (26).

\section{Local existence: proof of Theorem 2.1}

In the proof of Theorem 2.1, we employ the next result, which is a generalization to the non-linear case of Gronwall's lemma (cf. [4, Section 3]).

Lemma 5.1 (Bihari's inequality). Let $k$ be a nonnegative, continuous function, $M$ a real constant and $g$ a continuous, non-decreasing, nonnegative function such that

$$
G(u)=\int_{0}^{u} \frac{d s}{g(s)}
$$

is well-defined. Let $y$ be a continuous function such that

$$
y(t) \leq M+\int_{0}^{t} k(s) g(y(s)) d s
$$


for any $t \geq 0$. Then,

$$
G(y(t)) \leq G(M)+\int_{0}^{t} k(s) d s
$$

for any $t \geq 0$.

Using a standard contraction argument we prove now Theorem 2.1, following the main ideas of $[12$, Appendix A]. Note that differently from the global existence result, in this case we do not have to require a lower bound for the exponent $p$.

Proof of Theorem 2.1. Let $T, K$ be positive constants on which will be prescribed several conditions of suitability throughout this proof. We define

$$
B_{T, K}^{\psi} \doteq\left\{v \in \mathscr{C}\left([0, T], H^{1}\left(\mathbf{H}_{n}\right)\right) \cap \mathscr{C}^{1}\left([0, T], L^{2}\left(\mathbf{H}_{n}\right)\right):\|v\|_{T}^{\psi} \leq K\right\},
$$

where the norm $\|\cdot\|_{T}^{\psi}$ is defined by

$$
\|v\|_{T}^{\psi} \doteq \sup _{t \in[0, T]}\left(\left\|\mathrm{e}^{\psi(t, \cdot)} v(t, \cdot)\right\|_{L^{2}\left(\mathbf{H}_{n}\right)}+\left\|\mathrm{e}^{\psi(t, \cdot)} \nabla_{\mathrm{H}} v(t, \cdot)\right\|_{L^{2}\left(\mathbf{H}_{n}\right)}+\left\|\mathrm{e}^{\psi(t, \cdot)} v_{t}(t, \cdot)\right\|_{L^{2}\left(\mathbf{H}_{n}\right)}\right) .
$$

We introduce the map

$$
\begin{aligned}
\Phi: B_{T, K}^{\psi} & \longrightarrow \mathscr{C}\left([0, T], H^{1}\left(\mathbf{H}_{n}\right)\right) \cap \mathscr{C}^{1}\left([0, T], L^{2}\left(\mathbf{H}_{n}\right)\right), \\
v & \longmapsto u=\Phi(v),
\end{aligned}
$$

where $u$ solves the Cauchy problem

$$
\begin{cases}u_{t t}-\Delta_{\mathrm{H}} u+u_{t}=|v|^{p}, & (t, \eta) \in(0, T) \times \mathbf{H}_{n}, \\ u(0, \eta)=u_{0}(\eta), & \eta \in \mathbf{H}_{n} \\ u_{t}(0, \eta)=u_{1}(\eta), & \eta \in \mathbf{H}_{n} .\end{cases}
$$

We shall prove that, for a suitable choice of $T$ and $K, \Phi$ is a contraction map from $B_{T, K}^{\psi}$ to itself. From (15) it results

$$
\mathrm{e}^{2 \psi} u_{t}|v|^{p} \geq \frac{\partial}{\partial t}\left(\frac{\mathrm{e}^{2 \psi}}{2}\left(u_{t}^{2}+\left|\nabla_{\mathrm{H}} u\right|^{2}\right)\right)-\operatorname{div}\left(\mathrm{e}^{2 \psi} u_{t} \nabla_{\mathrm{H}} u\right) .
$$

So, introducing the weighted energy of the function $u$

$$
\mathscr{E}_{\psi}[u](t) \doteq \frac{1}{2} \int_{\mathbf{H}_{n}} \mathrm{e}^{2 \psi(t, \eta)}\left(\left|u_{t}(t, \eta)\right|^{2}+\left|\nabla_{\mathbf{H}} u(t, \eta)\right|^{2}\right) \mathrm{d} \eta
$$

and integrating over $[0, t] \times \mathbf{H}_{n}$ the last inequality, we have

$$
\mathscr{E}_{\psi}[u](t) \leq \mathscr{E}_{\psi}[u](0)+\int_{0}^{t} \int_{\mathbf{H}_{n}} \mathrm{e}^{2 \psi(s, \eta)} u_{t}(s, \eta)|v(s, \eta)|^{p} \mathrm{~d} \eta \mathrm{d} s,
$$

where we used the divergence theorem. Applying Cauchy-Schwarz inequality, we obtain

$$
\begin{aligned}
\mathscr{E}_{\psi}[u](t) & \leq \mathscr{E}_{\psi}[u](0)+\int_{0}^{t}\left(\int_{\mathbf{H}_{n}} \mathrm{e}^{2 \psi(s, \eta)}|v(s, \eta)|^{2 p} \mathrm{~d} \eta\right)^{\frac{1}{2}}\left(\int_{\mathbf{H}_{n}} \mathrm{e}^{2 \psi(s, \eta)}\left|u_{t}(s, \eta)\right|^{2} \mathrm{~d} \eta\right)^{\frac{1}{2}} \mathrm{~d} s \\
& \leq \mathscr{E}_{\psi}[u](0)+\sqrt{2} \int_{0}^{t}\left(\int_{\mathbf{H}_{n}} \mathrm{e}^{2 \psi(s, \eta)}|v(s, \eta)|^{2 p} \mathrm{~d} \eta\right)^{\frac{1}{2}} \mathscr{E}_{\psi}[u](s)^{\frac{1}{2}} \mathrm{~d} s .
\end{aligned}
$$


Thanks to Bihari's inequality, with $g(u)=(2 u)^{\frac{1}{2}}$, we find

$$
\mathscr{E}_{\psi}[u](t)^{\frac{1}{2}} \leq \mathscr{E}_{\psi}[u](0)^{\frac{1}{2}}+\frac{1}{\sqrt{2}} \int_{0}^{t}\left(\int_{\mathbf{H}_{n}} \mathrm{e}^{2 \psi(s, \eta)}|v(s, \eta)|^{2 p} \mathrm{~d} \eta\right)^{\frac{1}{2}} \mathrm{~d} s .
$$

The condition $v \in B_{T, K}^{\psi}$ implies $v(t, \cdot) \in H_{\psi(t, \cdot)}^{1}\left(\mathbf{H}_{n}\right)$ for any $t \in[0, T]$. Also, from Lemma 4.3 we get

$$
\begin{aligned}
\int_{\mathbf{H}_{n}} \mathrm{e}^{2 \psi(s, \eta)}|v(s, \eta)|^{2 p} \mathrm{~d} \eta & =\left\|\mathrm{e}^{\frac{1}{p} \psi(s, \cdot)} v(s, \cdot)\right\|_{L^{2 p}\left(\mathbf{H}_{n}\right)}^{2 p} \\
& \lesssim(1+s)^{p(1-\theta(2 p))}\left\|\nabla_{\mathrm{H}} v(s, \cdot)\right\|_{L^{2}\left(\mathbf{H}_{n}\right)}^{2(p-1)}\left\|\mathrm{e}^{\psi(s, \cdot)} \nabla_{\mathrm{H}} v(s, \cdot)\right\|_{L^{2}\left(\mathbf{H}_{n}\right)}^{2} \\
& \lesssim(1+s)^{p(1-\theta(2 p))}\left\|\mathrm{e}^{\psi(s, \cdot)} \nabla_{\mathrm{H}} v(s, \cdot)\right\|_{L^{2}\left(\mathbf{H}_{n}\right)}^{2 p} \\
& \leq(1+s)^{p(1-\theta(2 p))} K^{2 p} .
\end{aligned}
$$

Consequently, from (30) we have

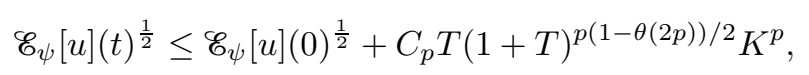

where $C_{p}>0$ is a multiplicative constant independent of $T$ and $K$ that may change from line to line up to the end of the proof. Therefore, we get

$$
\left\|\mathrm{e}^{\psi(t, \cdot)} u_{t}(t, \cdot)\right\|_{L^{2}\left(\mathbf{H}_{n}\right)}+\left\|\mathrm{e}^{\psi(t, \cdot)} \nabla_{\mathrm{H}} u(t, \cdot)\right\|_{L^{2}\left(\mathbf{H}_{n}\right)} \leq C_{p} \mathscr{E}_{\psi}[u](0)^{\frac{1}{2}}+C_{p} T(1+T)^{p(1-\theta(2 p)) / 2} K^{p} .
$$

On the other hand, since

$$
\mathrm{e}^{\psi(t, \eta)} u(t, \eta)=\mathrm{e}^{\psi(t, \eta)} u_{0}(\eta)+\int_{0}^{t} \mathrm{e}^{\psi(t, \eta)} u_{t}(s, \eta) \mathrm{d} s
$$

and $\psi$ is decreasing with respect to $t$, we have

$$
\begin{aligned}
\left\|\mathrm{e}^{\psi(t, \cdot)} u(t, \cdot)\right\|_{L^{2}\left(\mathbf{H}_{n}\right)} & \leq\left\|\mathrm{e}^{\psi(t, \cdot)} u_{0}\right\|_{L^{2}\left(\mathbf{H}_{n}\right)}+\int_{0}^{t}\left\|\mathrm{e}^{\psi(t, \cdot)} u_{t}(s, \cdot)\right\|_{L^{2}\left(\mathbf{H}_{n}\right)} \mathrm{d} s \\
& \leq\left\|\mathrm{e}^{\psi(t, \cdot)} u_{0}\right\|_{L^{2}\left(\mathbf{H}_{n}\right)}+\int_{0}^{t}\left\|\mathrm{e}^{\psi(s, \cdot)} u_{t}(s, \cdot)\right\|_{L^{2}\left(\mathbf{H}_{n}\right)} \mathrm{d} s \\
& \leq\left\|\mathrm{e}^{\psi(t, \cdot)} u_{0}\right\|_{L^{2}\left(\mathbf{H}_{n}\right)}+C_{p} \mathscr{\mathscr { E }}_{\psi}[u](0)^{\frac{1}{2}} T+C_{p} T^{2}(1+T)^{p(1-\theta(2 p)) / 2} K^{p},
\end{aligned}
$$

where in the last step we used (31). So, we have just proved that

$$
\begin{aligned}
\left\|\mathrm{e}^{\psi(t, \cdot)} u(t, \cdot)\right\|_{L^{2}\left(\mathbf{H}_{n}\right)}+ & \left\|\mathrm{e}^{\psi(t, \cdot)} u_{t}(t, \cdot)\right\|_{L^{2}\left(\mathbf{H}_{n}\right)}+\left\|\mathrm{e}^{\psi(t, \cdot)} \nabla_{\mathrm{H}} u(t, \cdot)\right\|_{L^{2}\left(\mathbf{H}_{n}\right)} \\
\leq & \left\|\mathrm{e}^{\psi(t, \cdot)} u_{0}\right\|_{L^{2}\left(\mathbf{H}_{n}\right)}+C_{p}(1+T) \mathscr{E}_{\psi}[u](0)^{\frac{1}{2}}+C_{p} T^{2}(1+T)^{p(1-\theta(2 p))} K^{p} \\
\leq & \left\|\mathrm{e}^{\psi(t, \cdot)} u_{0}\right\|_{L^{2}\left(\mathbf{H}_{n}\right)}+C_{p}(1+T)\left(\left\|\mathrm{e}^{\psi(t, \cdot)} \nabla_{\mathrm{H}} u_{0}\right\|_{L^{2}\left(\mathbf{H}_{n}\right)}+\left\|\mathrm{e}^{\psi(t, \cdot)} u_{1}\right\|_{L^{2}\left(\mathbf{H}_{n}\right)}\right) \\
& \quad+C_{p} T^{2}(1+T)^{p(1-\theta(2 p)) / 2} K^{p} .
\end{aligned}
$$

Clearly, we may take $K$ sufficiently large such that

$$
\frac{K}{2}>\left\|\mathrm{e}^{\psi(t, \cdot)} u_{0}\right\|_{L^{2}\left(\mathbf{H}_{n}\right)}+C_{p}\left(\left\|\mathrm{e}^{\psi(t, \cdot)} \nabla_{\mathrm{H}} u_{0}\right\|_{L^{2}\left(\mathbf{H}_{n}\right)}+\left\|\mathrm{e}^{\psi(t, \cdot)} u_{1}\right\|_{L^{2}\left(\mathbf{H}_{n}\right)}\right) .
$$

Hence, fixing now $T>0$ small enough so that

$$
\frac{K}{2} T+C_{p} T^{2}(1+T)^{p(1-\theta(2 p)) / 2} K^{p}<\frac{K}{2},
$$

since the above estimates are uniform in $t$, it follows that $\|v\|_{T}^{\psi} \leq K$, that is, $\Phi$ maps $B_{T, K}^{\psi}$ to itself. 
Finally, we have to prove that $\Phi$ is a contraction map, provided that $T$ is sufficiently small. Let us take $v, \bar{v} \in B_{T, K}^{\psi}$. If we denote $u \doteq \Phi(u), \bar{u} \doteq \Phi(\bar{v})$, then, $w=u-\bar{u}$ solves the Cauchy problem

$$
\begin{cases}w_{t t}-\Delta_{\mathrm{H}} w+w_{t}=|v|^{p}-|\bar{v}|^{p}, & (t, \eta) \in(0, T) \times \mathbf{H}_{n}, \\ u(0, \eta)=u_{t}(0, \eta)=0, & \eta \in \mathbf{H}_{n}\end{cases}
$$

Using again (15) and the divergence theorem, after integrating over $[0, t] \times \mathbf{H}_{n}$, we get the inequality

$$
\mathscr{E}_{\psi}[w](t) \leq \int_{0}^{t} \int_{\mathbf{H}_{n}} \mathrm{e}^{2 \psi(s, \eta)}\left(|v(s, \eta)|^{p}-|\bar{v}(s, \eta)|^{p}\right) w_{t}(s, \eta) \mathrm{d} \eta \mathrm{d} s .
$$

By $\left.|| v\right|^{p}-|\bar{v}|^{p}|\leq p| v-\bar{v} \mid(|v|+|\bar{v}|)^{p-1}$ and Cauchy-Schwarz inequality, we arrive at

$$
\begin{aligned}
\mathscr{E}_{\psi}[w] & (t) \lesssim \int_{0}^{t} \int_{\mathbf{H}_{n}} \mathrm{e}^{2 \psi(s, \eta)}|v(s, \eta)-\bar{v}(s, \eta)|(|v(s, \eta)|+|\bar{v}(s, \eta)|)^{p-1} w_{t}(s, \eta) \mathrm{d} \eta \mathrm{d} s \\
\leq & \int_{0}^{t}\left(\int_{\mathbf{H}_{n}} \mathrm{e}^{2 \psi(s, \eta)}\left|w_{t}(s, \eta)\right|^{2} \mathrm{~d} \eta\right)^{\frac{1}{2}}\left(\int_{\mathbf{H}_{n}} \mathrm{e}^{2 \psi(s, \eta)}|v(s, \eta)-\bar{v}(s, \eta)|^{2}(|v(s, \eta)|+|\bar{v}(s, \eta)|)^{2(p-1)} \mathrm{d} \eta\right)^{\frac{1}{2}} \mathrm{~d} s \\
& \leq \int_{0}^{t} \mathscr{E}_{\psi}[w](s)^{\frac{1}{2}}\left(\int_{\mathbf{H}_{n}} \mathrm{e}^{2 \psi(s, \eta)}|v(s, \eta)-\bar{v}(s, \eta)|^{2}(|v(s, \eta)|+|\bar{v}(s, \eta)|)^{2(p-1)} \mathrm{d} \eta\right)^{\frac{1}{2}} \mathrm{~d} s
\end{aligned}
$$

Applying again Lemma 5.1, we find the inequality

$$
\mathscr{E}_{\psi}[w](t)^{\frac{1}{2}} \lesssim \int_{0}^{t}\left(\int_{\mathbf{H}_{n}} \mathrm{e}^{2 \psi(s, \eta)}|v(s, \eta)-\bar{v}(s, \eta)|^{2}(|v(s, \eta)|+|\bar{v}(s, \eta)|)^{2(p-1)} \mathrm{d} \eta\right)^{\frac{1}{2}} \mathrm{~d} s .
$$

By Hölder's inequality it follows

$$
\begin{aligned}
& \left\|\mathrm{e}^{\psi(s, \cdot)}|v(s, \cdot)-\bar{v}(s, \cdot)|(|v(s, \cdot)|+|\bar{v}(s, \cdot)|)^{p-1}\right\|_{L^{2}\left(\mathbf{H}_{n}\right)} \\
& \quad \leq\left\|\mathrm{e}^{(2-p) \psi(s, \cdot)}|v(s, \cdot)-\bar{v}(s, \cdot)|\right\|_{L^{2 p}\left(\mathbf{H}_{n}\right)}\left\|\mathrm{e}^{(p-1) \psi(s, \cdot)}(|v(s, \cdot)|+|\bar{v}(s, \cdot)|)^{p-1}\right\|_{L^{\frac{2 p}{p-1}}\left(\mathbf{H}_{n}\right)} .
\end{aligned}
$$

We estimate separately the two norms on the right-hand side of the last inequality. Using Lemma 4.3 and the property $\psi \geq 0$, we get

$$
\left\|\mathrm{e}^{(2-p) \psi(s, \cdot)}|v(s, \cdot)-\bar{v}(s, \cdot)|\right\|_{L^{2 p}\left(\mathbf{H}_{n}\right)} \lesssim(1+s)^{(1-\theta(2 p)) / 2}\left\|\mathrm{e}^{\psi(s, \cdot)} \nabla_{\mathrm{H}}(v(s, \cdot)-\bar{v}(s, \cdot))\right\|_{L^{2}\left(\mathbf{H}_{n}\right)}
$$

and

$$
\begin{aligned}
\left\|\mathrm{e}^{(p-1) \psi(s, \cdot)}(|v(s, \cdot)|+|\bar{v}(s, \cdot)|)^{p-1}\right\|_{L^{\frac{2 p}{p-1}}\left(\mathbf{H}_{n}\right)}=\left(\int_{\mathbf{H}_{n}} \mathrm{e}^{2 p \psi(s, \eta)}(|v(s, \eta)|+|\bar{v}(s, \eta)|)^{2 p} \mathrm{~d} \eta\right)^{\frac{p-1}{2 p}} \\
\quad \lesssim\left(\left\|\mathrm{e}^{\psi(s, \cdot)} v(s, \cdot)\right\|_{L^{2 p}\left(\mathbf{H}_{n}\right)}+\left\|\mathrm{e}^{\psi(s, \cdot)} \bar{v}(s, \cdot)\right\|_{L^{2 p}\left(\mathbf{H}_{n}\right)}\right)^{p-1} \\
\quad \lesssim(1+s)^{(1-\theta(2 p))(p-1) / 2}\left(\left\|\mathrm{e}^{\psi(s, \cdot)} \nabla_{\mathrm{H}} v(s, \cdot)\right\|_{L^{2}\left(\mathbf{H}_{n}\right)}+\left\|\mathrm{e}^{\psi(s, \cdot)} \nabla_{\mathrm{H}} \bar{v}(s, \cdot)\right\|_{L^{2}\left(\mathbf{H}_{n}\right)}\right)^{p-1} .
\end{aligned}
$$

By (32) we have

$$
\begin{aligned}
\left\|\mathrm{e}^{\psi(t, \cdot)} w_{t}(t, \cdot)\right\|_{L^{2}\left(\mathbf{H}_{n}\right)} & +\left\|\mathrm{e}^{\psi(t, \cdot)} \nabla_{\mathrm{H}} w(t, \cdot)\right\|_{L^{2}\left(\mathbf{H}_{n}\right)} \\
\leq & C_{p} \int_{0}^{t}(1+s)^{p(1-\theta(2 p)) / 2}\left\|\mathrm{e}^{\psi(s, \cdot)} \nabla_{\mathrm{H}}(v(s, \cdot)-\bar{v}(s, \cdot))\right\|_{L^{2}\left(\mathbf{H}_{n}\right)} \\
& \quad \times\left(\left\|\mathrm{e}^{\psi(s, \cdot)} \nabla_{\mathrm{H}} v(s, \cdot)\right\|_{L^{2}\left(\mathbf{H}_{n}\right)}+\left\|\mathrm{e}^{\psi(s, \cdot)} \nabla_{\mathrm{H}} \bar{v}(s, \cdot)\right\|_{L^{2}\left(\mathbf{H}_{n}\right)}\right)^{p-1} \mathrm{~d} s \\
& \leq C_{p} \int_{0}^{t}(1+s)^{p(1-\theta(2 p)) / 2} \mathrm{~d} s\|v-\bar{v}\|_{T}^{\psi}\left(\|v\|_{T}^{\psi}+\|\bar{v}\|_{T}^{\psi}\right)^{p-1} \\
& \leq C_{p} T(1+T)^{p(1-\theta(2 p)) / 2} K^{p-1}\|v-\bar{v}\|_{T}^{\psi} .
\end{aligned}
$$


Furthermore,

$$
\mathrm{e}^{\psi(t, \eta)} w(t, \eta)=\int_{0}^{t} \mathrm{e}^{\psi(t, \eta)} w_{t}(s, \eta) \mathrm{d} s
$$

and the fact that $\psi$ is decreasing with respect to $t$ imply

$$
\begin{aligned}
\left\|\mathrm{e}^{\psi(t, \cdot)} w(t, \cdot)\right\|_{L^{2}\left(\mathbf{H}_{n}\right)} & \leq \int_{0}^{t}\left\|\mathrm{e}^{\psi(t, \eta)} w_{t}(s, \eta)\right\|_{L^{2}\left(\mathbf{H}_{n}\right)} \mathrm{d} s \leq \int_{0}^{t}\left\|\mathrm{e}^{\psi(s, \eta)} w_{t}(s, \eta)\right\|_{L^{2}\left(\mathbf{H}_{n}\right)} \mathrm{d} s \\
& \leq C_{p} T^{2}(1+T)^{p(1-\theta(2 p)) / 2} K^{p-1}\|v-\bar{v}\|_{T}^{\psi},
\end{aligned}
$$

where in the last inequality we applied (33). Summarizing, combining (33) and (34) we arrive at

$$
\|\Phi(v)-\Phi(\bar{v})\|_{T}^{\psi}=\|w\|_{T}^{\psi} \leq C_{p} T(1+T)^{1+p(1-\theta(2 p)) / 2} K^{p-1}\|v-\bar{v}\|_{T}^{\psi} .
$$

So, choosing $T>0$ sufficiently small we find that $\Phi$ is a contraction.

Therefore, our starting problem has a unique solution $u$ in $\mathscr{C}\left(\left[0, T_{\max }\right), H^{1}\left(\mathbf{H}_{n}\right)\right) \cap \mathscr{C}^{1}\left(\left[0, T_{\max }\right), L^{2}\left(\mathbf{H}_{n}\right)\right)$ with finite energy $\mathscr{E}_{\psi}[u](t)$ for any $t \in\left[0, T_{\max }\right)$, due to Banach's fixed point theorem. Moreover $T_{\max }<\infty$ implies the blow up of the energy for $T \rightarrow T_{\max }^{-}$. Otherwise, if it was not so, we would have a finite energy for $u$ in a left neighborhood of $T_{\max }$, and then repeating the same arguments when the initial conditions are taken for $t=0$, we could extend the solution, violating the maximality of $T_{\max }$.

\section{Estimates for the linear problem}

In order to prove Theorem 2.2, we recall some decay estimates for the solution of the linear Cauchy problem (10). In the next propositions we can relax the assumptions for the initial data, considering a less restrictive space than the weighted energy space $\mathcal{A}\left(\mathbf{H}_{n}\right)$. More precisely, we may assume just data in the classical energy spaces with additional $L^{1}\left(\mathbf{H}_{n}\right)$ regularity, namely,

$$
\left(u_{0}, u_{1}\right) \in\left(H^{1}\left(\mathbf{H}_{n}\right) \cap L^{1}\left(\mathbf{H}_{n}\right)\right) \times\left(L^{2}\left(\mathbf{H}_{n}\right) \cap L^{1}\left(\mathbf{H}_{n}\right)\right) .
$$

We set

$$
\mathscr{D}^{\kappa}\left(\mathbf{H}_{n}\right) \doteq\left(H^{\kappa}\left(\mathbf{H}_{n}\right) \cap L^{1}\left(\mathbf{H}_{n}\right)\right) \times\left(L^{2}\left(\mathbf{H}_{n}\right) \cap L^{1}\left(\mathbf{H}_{n}\right)\right) \quad \text { for } \kappa \in\{0,1\}
$$

Clearly,

$$
\mathscr{A}\left(\mathbf{H}_{n}\right) \hookrightarrow \mathscr{D}_{1}^{1}\left(\mathbf{H}_{n}\right) \hookrightarrow H^{1}\left(\mathbf{H}_{n}\right) \times L^{2}\left(\mathbf{H}_{n}\right) .
$$

Proposition 6.1. Let us assume $\left(u_{0}, u_{1}\right) \in \mathscr{D}^{1}\left(\mathbf{H}_{n}\right)$. Let $u \in \mathscr{C}\left([0, \infty), H^{1}\left(\mathbf{H}_{n}\right)\right) \cap \mathscr{C}^{1}\left([0, \infty), L^{2}\left(\mathbf{H}_{n}\right)\right)$ solve the Cauchy problem (10). Then, the following decay estimates are satisfied

$$
\begin{aligned}
\|u(t, \cdot)\|_{L^{2}\left(\mathbf{H}_{\mathbf{n}}\right)} & \leq C(1+t)^{-\frac{\varrho}{4}}\left\|\left(u_{0}, u_{1}\right)\right\|_{\mathscr{D}^{0}\left(\mathbf{H}_{n}\right)} \\
\left\|\nabla_{\mathrm{H}} u(t, \cdot)\right\|_{L^{2}\left(\mathbf{H}_{\mathbf{n}}\right)} & \leq C(1+t)^{-\frac{\varrho}{4}-\frac{1}{2}}\left\|\left(u_{0}, u_{1}\right)\right\|_{\mathscr{D}^{1}\left(\mathbf{H}_{n}\right)} \\
\left\|\partial_{t} u(t, \cdot)\right\|_{L^{2}\left(\mathbf{H}_{\mathbf{n}}\right)} & \leq C(1+t)^{-\frac{\varrho}{4}-1}\left\|\left(u_{0}, u_{1}\right)\right\|_{\mathscr{D}^{1}\left(\mathbf{H}_{n}\right)}
\end{aligned}
$$

for any $t \geq 0$. Furthermore, if we assume just $\left(u_{0}, u_{1}\right) \in H^{1}\left(\mathbf{H}_{n}\right) \times L^{2}\left(\mathbf{H}_{n}\right)$, that is, we do not require additional $L^{1}\left(\mathbf{H}_{n}\right)$ regularity for the Cauchy data, then the following estimates are satisfied

$$
\begin{aligned}
\|u(t, \cdot)\|_{L^{2}\left(\mathbf{H}_{\mathbf{n}}\right)} & \leq C\left\|\left(u_{0}, u_{1}\right)\right\|_{L^{2}\left(\mathbf{H}_{n}\right)} \\
\left\|\nabla_{\mathrm{H}} u(t, \cdot)\right\|_{L^{2}\left(\mathbf{H}_{\mathbf{n}}\right)} & \leq C(1+t)^{-\frac{1}{2}}\left\|\left(u_{0}, u_{1}\right)\right\|_{H^{1}\left(\mathbf{H}_{n}\right) \times L^{2}\left(\mathbf{H}_{n}\right)} \\
\left\|\partial_{t} u(t, \cdot)\right\|_{L^{2}\left(\mathbf{H}_{\mathbf{n}}\right)} & \leq C(1+t)^{-1}\left\|\left(u_{0}, u_{1}\right)\right\|_{H^{1}\left(\mathbf{H}_{n}\right) \times L^{2}\left(\mathbf{H}_{n}\right)}
\end{aligned}
$$

for any $t \geq 0$. Here $C>0$ is a universal constant.

Proof. See [17, Theorem 1.1], where the group Fourier transform on $\mathbf{H}_{n}$ is applied to prove this result.

Finally, let us point out explicitly that we can still employ the estimates derived in the previous proposition in order to estimate Duhamel's integral term (11), as the operator $\partial_{t}^{2}-\Delta_{\mathrm{H}}+\partial_{t}$ is invariant by time translations. 


\section{Global existence of small data solutions: proof of Theorem 2.2}

In order to prove Theorem 2.2, first we have to prove the next preliminary lemma, which allows us to estimate the weighted energy (29) of a local (in time) solution $u$ to (1).

Lemma 7.1. Let $n \geq 1$ and $p>1$ such that $p \leq \frac{\mathbb{Q}}{\mathbb{Q}-2}$. Let $\left(u_{0}, u_{1}\right) \in \mathscr{A}\left(\mathbf{H}_{n}\right)$. If $u$ solves

$$
\begin{cases}u_{t t}-\Delta_{\mathrm{H}} u+u_{t}=|u|^{p}, & (t, \eta) \in(0, T) \times \mathbf{H}_{n}, \\ u(0, \eta)=u_{0}(\eta), & \eta \in \mathbf{H}_{n} \\ u_{t}(0, \eta)=u_{1}(\eta), & \eta \in \mathbf{H}_{n},\end{cases}
$$

then, the following energy estimate holds for any $t \in[0, T)$ and for an arbitrary small $\delta>0$

$$
\mathscr{E}_{\psi}[u](t) \lesssim I_{0}^{2}+I_{0}^{p+1}+\left(\sup _{s \in[0, t]}(1+s)^{\delta}\left\|\mathrm{e}^{\left(\frac{2}{p+1}+\delta\right) \psi(s, \cdot)} u(s, \cdot)\right\|_{L^{p+1}\left(\mathbf{H}_{n}\right)}\right)^{p+1}
$$

where

$$
I_{0}^{2} \doteq \int_{\mathbf{H}_{n}} \mathrm{e}^{2 \psi(0, \eta)}\left(\left|u_{1}(\eta)\right|^{2}+\left|\nabla_{\mathrm{H}} u_{0}(\eta)\right|^{2}\right) \mathrm{d} \eta
$$

Proof. First we prove that

$$
\mathscr{E}_{\psi}[u](t) \lesssim I_{0}^{2}+I_{0}^{p+1}+\left\|\mathrm{e}^{\frac{2}{p+1} \psi(t, \cdot)} u(t, \cdot)\right\|_{L^{p+1}\left(\mathbf{H}_{n}\right)}^{p+1}+\int_{0}^{t} \int_{\mathbf{H}_{n}}\left|\psi_{t}(s, \eta)\right| \mathrm{e}^{2 \psi(s, \eta)}|u(s, \eta)|^{p+1} \mathrm{~d} \eta \mathrm{d} s .
$$

Integrating the relation (21) over $[0, t] \times \mathbf{H}_{n}$, we get immediately (after using the divergence theorem)

$$
\mathscr{G}_{\psi}[u](t) \leq \mathscr{G}_{\psi}[u](0)-\frac{2}{p+1} \int_{0}^{t} \int_{\mathbf{H}_{n}} \psi_{t}(s, \eta) \mathrm{e}^{2 \psi(s, \eta)}|u(s, \eta)|^{p} u(s, \eta) \mathrm{d} \eta \mathrm{d} s,
$$

where

$$
\mathscr{G}_{\psi}[u](t) \doteq \mathscr{E}_{\psi}[u](t)-\frac{1}{p+1} \int_{\mathbf{H}_{n}} \mathrm{e}^{2 \psi(t, \eta)}|u(t, \eta)|^{p} u(t, \eta) \mathrm{d} \eta
$$

Consequently,

$$
\begin{aligned}
& \mathscr{E}_{\psi}[u](t) \leq \mathscr{G}_{\psi}[u](0)+\frac{1}{p+1} \int_{\mathbf{H}_{n}} \mathrm{e}^{2 \psi(t, \eta)}|u(t, \eta)|^{p} u(t, \eta) \mathrm{d} \eta-\frac{2}{p+1} \int_{0}^{t} \int_{\mathbf{H}_{n}} \psi_{t}(s, \eta) \mathrm{e}^{2 \psi(s, \eta)}|u(s, \eta)|^{p} u(s, \eta) \mathrm{d} \eta \mathrm{d} s \\
& \lesssim \mathscr{G}_{\psi}[u](0)+\left\|\mathrm{e}^{\frac{2}{p+1} \psi(t, \cdot)} u(t, \cdot)\right\|_{L^{p+1}\left(\mathbf{H}_{n}\right)}^{p+1}+\int_{0}^{t} \int_{\mathbf{H}_{n}}\left|\psi_{t}(s, \eta)\right| \mathrm{e}^{2 \psi(s, \eta)}|u(s, \eta)|^{p+1} \mathrm{~d} \eta \mathrm{d} s .
\end{aligned}
$$

So, in order to prove $(43)$ we have just to show that $\mathscr{G}_{\psi}[u](0) \lesssim I_{0}^{2}+I_{0}^{p+1}$. Since

$$
\mathscr{G}_{\psi}[u](0)=\mathscr{E}_{\psi}[u](0)-\frac{1}{p+1} \int_{\mathbf{H}_{n}} \mathrm{e}^{\psi(0, \eta)}\left|u_{0}(\eta)\right|^{p} u_{0}(\eta) \mathrm{d} \eta \lesssim I_{0}^{2}+\int_{\mathbf{H}_{n}} \mathrm{e}^{\psi(0, \eta)}\left|u_{0}(\eta)\right|^{p+1} \mathrm{~d} \eta
$$

we have to prove only that

$$
\int_{\mathbf{H}_{n}} \mathrm{e}^{\psi(0, \eta)}\left|u_{0}(\eta)\right|^{p+1} \mathrm{~d} \eta \lesssim I_{0}^{p+1}
$$

Because of $p+1<\frac{\mathbb{Q}}{\mathbb{Q}-2}+1<\frac{2 \mathbb{Q}}{\mathbb{Q}-2}$, using the Sobolev embedding

$$
H^{1}\left(\mathbf{H}_{n}\right) \hookrightarrow L^{p+1}\left(\mathbf{H}_{n}\right)
$$


which follows, for example, from the special case $\theta=1$ in Lemma 4.1 by interpolation with the trivial embedding $H^{1}\left(\mathbf{H}_{n}\right) \hookrightarrow L^{2}\left(\mathbf{H}_{n}\right)$, we find

$$
\begin{aligned}
\int_{\mathbf{H}_{n}} \mathrm{e}^{\psi(0, \eta)}\left|u_{0}(\eta)\right|^{p+1} \mathrm{~d} \eta & =\left\|\mathrm{e}^{\frac{1}{p+1} \psi(0, \cdot)} u_{0}\right\|_{L^{p+1}\left(\mathbf{H}_{n}\right)}^{p+1} \lesssim\left\|\mathrm{e}^{\frac{1}{p+1} \psi(0, \cdot)} u_{0}\right\|_{H^{1}\left(\mathbf{H}_{n}\right)}^{p+1} \\
& =\left(\int_{\mathbf{H}_{n}} \mathrm{e}^{\frac{2}{p+1} \psi(0, \eta)}\left(\left|u_{0}(\eta)\right|^{2}+\left|\nabla_{\mathrm{H}} u_{0}(\eta)\right|^{2}+(p+1)^{-2}\left|\nabla_{\mathrm{H}} \psi(0, \eta)\right|^{2}\left|u_{0}(\eta)\right|^{2}\right) \mathrm{d} \eta\right)^{\frac{p+1}{2}} \\
& \lesssim\left(\int_{\mathbf{H}_{n}} \mathrm{e}^{\frac{2}{p+1} \psi(0, \eta)}\left(\left|u_{0}(\eta)\right|^{2}+\left|\nabla_{\mathrm{H}} u_{0}(\eta)\right|^{2}+\left(|x|^{2}+|y|^{2}\right)\left|u_{0}(\eta)\right|^{2}\right) \mathrm{d} \eta\right)^{\frac{p+1}{2}} \\
& \lesssim\left(\int_{\mathbf{H}_{n}} \mathrm{e}^{2 \psi(0, \eta)}\left(\left|u_{0}(\eta)\right|^{2}+\left|\nabla_{\mathbf{H}} u_{0}(\eta)\right|^{2}\right) \mathrm{d} \eta\right)^{\frac{p+1}{2}} \lesssim I_{0}^{p+1}
\end{aligned}
$$

where $\eta=(x, y, \tau)$ and in the second last inequality we have used the fact that $p>1$ to get the estimate

$$
\left(1+\left(|x|^{2}+|y|^{2}\right)\right) \mathrm{e}^{\frac{2}{p+1} \psi(0, \eta)} \lesssim \mathrm{e}^{2 \psi(0, \eta)} .
$$

So, we proved (43). From the relation $\psi_{t}(s, \eta)=-(1+s)^{-1} \psi(s, \eta)$ it follows

$$
\left|\psi_{t}(s, \eta)\right| \mathrm{e}^{(2-\gamma(p+1)) \psi(s, \eta)}=\frac{1}{1+s} \psi(s, \eta) \mathrm{e}^{-\delta(p+1) \psi(s, \eta)} \lesssim(1+s)^{-1},
$$

with $\gamma=\frac{2}{p+1}+\delta$ and $\delta>0$. Therefore,

$$
\begin{aligned}
\int_{0}^{t} \int_{\mathbf{H}_{n}}\left|\psi_{t}(s, \eta)\right| \mathrm{e}^{2 \psi(s, \eta)}|u(s, \eta)|^{p+1} \mathrm{~d} x \mathrm{~d} s & \lesssim \int_{0}^{t}(1+s)^{-1} \int_{\mathbf{H}_{n}} \mathrm{e}^{\gamma(p+1) \psi(s, \eta)}|u(s, \eta)|^{p+1} \mathrm{~d} \eta \mathrm{d} s \\
& \leq \sup _{s \in[0, t]}(1+s)^{\delta(p+1)}\left\|\mathrm{e}^{\gamma \psi(s, \cdot)} u(s, \cdot)\right\|_{L^{p+1}\left(\mathbf{H}_{n}\right)}^{p+1} \int_{0}^{t}(1+s)^{-1-\delta(p+1)} \mathrm{d} s \\
& \lesssim\left(\sup _{s \in[0, t]}(1+s)^{\delta}\left\|\mathrm{e}^{\gamma \psi(s, \cdot)} u(s, \cdot)\right\|_{L^{p+1}\left(\mathbf{H}_{n}\right)}\right)^{p+1}
\end{aligned}
$$

Finally, since $\gamma>\frac{2}{p+1}$ and $\delta>0$, we have trivially

$$
\left\|\mathrm{e}^{\frac{2}{p+1} \psi(t, \cdot)} u(t, \cdot)\right\|_{L^{p+1}\left(\mathbf{H}_{n}\right)}^{p+1} \leq\left((1+t)^{\delta}\left\|\mathrm{e}^{\gamma \psi(t, \cdot)} u(t, \cdot)\right\|_{L^{p+1}\left(\mathbf{H}_{n}\right)}\right)^{p+1} .
$$

Hence, combining (45), (43) and (44), we get the desired estimate (42).

Combing the linear estimates from Section 6 and Lemma 7.1, we can finally prove Theorem 2.2.

Proof of Theorem 2.2. By contradiction, let us assume that for any $\varepsilon_{0}>0$ there exists data satisfying (6) such that the solution $u \in \mathscr{C}\left(\left[0, T_{\max }\right), H_{\psi(t, \cdot)}^{1}\left(\mathbf{H}_{n}\right)\right) \cap \mathscr{C}^{1}\left(\left[0, T_{\max }\right), L_{\psi(t, \cdot)}^{2}\left(\mathbf{H}_{n}\right)\right)$ to the corresponding problem, whose existence is guaranteed by Theorem 2.1, is not global in time, that means $T_{\max }<\infty$.

For any $T \in\left(0, T_{\max }\right)$, we may define the Banach space

$$
X(T) \doteq \mathscr{b}\left([0, T], H_{\psi(t, \cdot)}^{1}\left(\mathbf{H}_{n}\right)\right) \cap \mathscr{C}^{1}\left([0, T], L_{\psi(t, \cdot)}^{2}\left(\mathbf{H}_{n}\right)\right),
$$

equipped with the norm

$$
\begin{gathered}
\|u\|_{X(T)} \doteq \sup _{t \in[0, T]}\left[\left\|\mathrm{e}^{\psi(t, \cdot)} \nabla_{\mathrm{H}} u(t, \cdot)\right\|_{L^{2}\left(\mathbf{H}_{n}\right)}+\left\|\mathrm{e}^{\psi(t, \cdot)} u_{t}(t, \cdot)\right\|_{L^{2}\left(\mathbf{H}_{n}\right)}+(1+t)^{\frac{6}{4}}\|u(t, \cdot)\|_{L^{2}\left(\mathbf{H}_{n}\right)}\right. \\
\left.\left.+(1+t)^{\frac{6}{4}+\frac{1}{2}}\left\|\nabla_{\mathrm{H}} u(t, \cdot)\right\|_{L^{2}\left(\mathbf{H}_{n}\right)}+(1+t)^{\frac{6}{4}+1}\left\|u_{t}(t, \cdot)\right\|_{L^{2}\left(\mathbf{H}_{n}\right)}\right)\right] .
\end{gathered}
$$


By Lemma 7.1 it follows that

$$
\begin{aligned}
\left\|\mathrm{e}^{\psi(t, \cdot)} u_{t}(t, \cdot)\right\|_{L^{2}\left(\mathbf{H}_{n}\right)} & +\left\|\mathrm{e}^{\psi(t, \cdot)} \nabla_{\mathrm{H}} u(t, \cdot)\right\|_{L^{2}\left(\mathbf{H}_{n}\right)} \\
& \lesssim \varepsilon_{0}+\varepsilon_{0}^{\frac{p+1}{2}}+\left(\sup _{s \in[0, t]}(1+s)^{\delta}\left\|\mathrm{e}^{\left(\delta+\frac{2}{p+1}\right) \psi(s, \cdot)} u(s, \cdot)\right\|_{L^{p+1}\left(\mathbf{H}_{n}\right)}\right)^{\frac{p+1}{2}} .
\end{aligned}
$$

As $2<p+1$ and $p+1<2 p \leq \frac{2 \mathbb{Q}}{\mathbb{Q}-2}$, we find that $\theta(p+1) \in(0,1]$. Besides, we may take $\delta>0$ sufficiently small such that $\delta+\frac{2}{p+1}<1$. Let us stress that throughout the proof we will prescribe further conditions that the quantity $\delta$ has to fulfill. Hence, by Lemma 4.3 we obtain

$$
\begin{aligned}
\left\|\mathrm{e}^{\left(\delta+\frac{2}{p+1}\right) \psi(s, \cdot)} u(s, \cdot)\right\|_{L^{p+1}\left(\mathbf{H}_{n}\right)} & \lesssim(1+s)^{\frac{1}{2}(1-\theta(p+1))}\left\|\nabla_{\mathrm{H}} u(s, \cdot)\right\|_{L^{2}\left(\mathbf{H}_{n}\right)}^{1-\left(\delta+\frac{2}{p+1}\right)}\left\|\mathrm{e}^{\psi(s, \cdot)} \nabla_{\mathrm{H}} u(s, \cdot)\right\|_{L^{2}\left(\mathbf{H}_{n}\right)}^{\delta+\frac{2}{2+1}} \\
& \lesssim(1+s)^{\frac{1}{2}(1-\theta(p+1))-\left(1-\left(\delta+\frac{2}{p+1}\right)\right)\left(\frac{\Theta}{4}+\frac{1}{2}\right)}\|u\|_{X(t)} \\
& \lesssim(1+s)^{\frac{\varrho+1}{p+1}-\frac{\Theta}{2}+\delta\left(\frac{\Theta}{4}+\frac{1}{2}\right)}\|u\|_{X(t)}
\end{aligned}
$$

for any $s \in[0, t]$. As we assume $p>p_{\text {Fuj }}(\mathbb{Q})$ (which is equivalent to require that $\frac{\mathbb{Q}+1}{p+1}-\frac{\mathbb{Q}}{2}<0$ ), we may consider $\delta>0$ such that

$$
\frac{Q+1}{p+1}-\frac{Q}{2}+\delta\left(\frac{Q}{4}+\frac{1}{2}+1\right)<0
$$

Therefore, by (46) we have

$$
\left\|\mathrm{e}^{\psi(t, \cdot)} u_{t}(t, \cdot)\right\|_{L^{2}\left(\mathbf{H}_{n}\right)}+\left\|\mathrm{e}^{\psi(t, \cdot)} \nabla_{\mathrm{H}} u(t, \cdot)\right\|_{L^{2}\left(\mathbf{H}_{n}\right)} \lesssim \varepsilon_{0}+\varepsilon_{0}^{\frac{p+1}{2}}+\|u\|_{X(t)}^{\frac{p+1}{2}} .
$$

Let us proceed now with the estimate of the not-weighted $L^{2}\left(\mathbf{H}_{\mathbf{n}}\right)$ - norms. We will follow precisely the computations for the Euclidean case (cf. [9, Section 18.1]). Thus,

$$
\begin{aligned}
\left\|\partial_{t}^{\ell} \nabla_{\mathrm{H}}^{k} u(t, \cdot)\right\|_{L^{2}\left(\mathbf{H}_{n}\right) \lesssim} & \varepsilon_{0}(1+t)^{-\frac{Q}{4}-\frac{k}{2}-\ell}+\int_{0}^{t / 2}(1+t-s)^{-\frac{Q}{4}-\frac{k}{2}-\ell}\left(\|u(s, \cdot)\|_{L^{p}\left(\mathbf{H}_{n}\right)}^{p}+\|u(s, \cdot)\|_{L^{2 p}\left(\mathbf{H}_{n}\right)}^{p}\right) \mathrm{d} s \\
& +\int_{t / 2}^{t}(1+t-s)^{-\frac{k}{2}-\ell}\|u(s, \cdot)\|_{L^{2 p}\left(\mathbf{H}_{n}\right)}^{p} \mathrm{~d} s
\end{aligned}
$$

for $k+\ell=0,1$, where we used (35) to estimate the solution of the corresponding linear homogeneous problem, the $L^{1} \cap L^{2}-L^{2}$ estimates (36), (37) and (38) to estimate Duhamel's term on the interval [0,t/2] and the $L^{2}-L^{2}$ estimates (39), (40) and (41) on the interval $[t / 2, t]$. Applying (7) and (8) to $|u(s, \cdot)|^{p}$ with $\sigma=\delta p$ and using $(25)$ and the definition of the norm $\|\cdot\|_{X(t)}$, we arrive at

$$
\begin{aligned}
\|u(s, \cdot)\|_{L^{p}\left(\mathbf{H}_{n}\right)}^{p} & \lesssim(1+s)^{\frac{\varrho}{4}}\left\|\mathrm{e}^{\delta \psi(s, \cdot)} u(s, \cdot)\right\|_{L^{2 p}\left(\mathbf{H}_{n}\right)}^{p} \\
& \lesssim(1+s)^{\frac{\varrho}{4}+\frac{p}{2}(1-\theta(2 p))}\left\|\nabla_{\mathrm{H}} u(s, \cdot)\right\|_{L^{2}\left(\mathbf{H}_{n}\right)}^{(1-\delta) p}\left\|\mathrm{e}^{\psi(s, \cdot)} \nabla_{\mathrm{H}} u(s, \cdot)\right\|_{L^{2}\left(\mathbf{H}_{n}\right)}^{\delta p} \\
& \lesssim(1+s)^{\frac{6}{4}+\frac{p}{2}(1-\theta(2 p))-(1-\delta) p\left(\frac{\Omega}{4}+\frac{1}{2}\right)}\|u\|_{X(t)}^{p}=(1+s)^{-\frac{\varrho p}{2}+\frac{\varrho}{2}+\delta p\left(\frac{\Omega}{4}+\frac{1}{2}\right)}\|u\|_{X(t)}^{p}
\end{aligned}
$$

and

$$
\begin{aligned}
\|u(s, \cdot)\|_{L^{2 p}\left(\mathbf{H}_{n}\right)}^{p} & \lesssim\left\|\mathrm{e}^{\delta \psi(s, \cdot)} u(s, \cdot)\right\|_{L^{2 p}\left(\mathbf{H}_{n}\right)}^{p} \\
& \lesssim(1+s)^{\frac{p}{2}(1-\theta(2 p))}\left\|\nabla_{\mathrm{H}} u(s, \cdot)\right\|_{L^{2}\left(\mathbf{H}_{n}\right)}^{(1-\delta) p}\left\|\mathrm{e}^{\psi(s, \cdot)} \nabla_{\mathrm{H}} u(s, \cdot)\right\|_{L^{2}\left(\mathbf{H}_{n}\right)}^{\delta p} \\
& \lesssim(1+s)^{\frac{p}{2}(1-\theta(2 p))-(1-\delta) p\left(\frac{\varrho}{4}+\frac{1}{2}\right)}\|u\|_{X(t)}^{p}=(1+s)^{-\frac{\varrho p}{2}+\frac{\varrho}{4}+\delta p\left(\frac{Q}{4}+\frac{1}{2}\right)}\|u\|_{X(t)}^{p},
\end{aligned}
$$

where we might apply (25) thanks to the upper bound $p \leq p_{\mathrm{GN}}(\mathbb{Q})$ that guarantees $\theta(2 p) \in(0,1]$. We estimate separately the two integrals on the right-hand side of (48). 
Let us begin with the integral over $[0, t / 2]$ :

$$
\begin{aligned}
& \int_{0}^{t / 2}(1+t-s)^{-\frac{\Omega}{4}-\frac{k}{2}-\ell}\left(\|u(s, \cdot)\|_{L^{p}\left(\mathbf{H}_{n}\right)}^{p}+\|u(s, \cdot)\|_{L^{2 p}\left(\mathbf{H}_{n}\right)}^{p}\right) \mathrm{d} s \\
& \lesssim \int_{0}^{t / 2}(1+t-s)^{-\frac{\Theta}{4}-\frac{k}{2}-\ell}(1+s)^{-\frac{\Theta p}{2}+\frac{\varrho}{2}+\delta p\left(\frac{\Theta}{4}+\frac{1}{2}\right)} \mathrm{d} s\|u\|_{X(t)}^{p} \\
& \lesssim(1+t)^{-\frac{\varrho}{4}-\frac{k}{2}-\ell} \int_{0}^{t / 2}(1+s)^{-\frac{\Theta p}{2}+\frac{\varrho}{2}+\delta p\left(\frac{\varrho}{4}+\frac{1}{2}\right)} \mathrm{d} s\|u\|_{X(t)}^{p} .
\end{aligned}
$$

Since $p>p_{\text {Fuj }}(\mathbb{Q})$ and, equivalently, $-\frac{Q p}{2}+\frac{Q}{2}<-1$, we can find $\delta>0$ such that

$$
-\frac{Q p}{2}+\frac{Q}{2}+\delta p\left(\frac{Q}{4}+\frac{1}{2}\right)<-1 .
$$

Consequently,

$$
\int_{0}^{t / 2}(1+t-s)^{-\frac{\varrho}{4}-\frac{k}{2}-\ell}\left(\|u(s, \cdot)\|_{L^{p}\left(\mathbf{H}_{n}\right)}^{p}+\|u(s, \cdot)\|_{L^{2 p}\left(\mathbf{H}_{n}\right)}^{p}\right) \mathrm{d} s \lesssim(1+t)^{-\frac{\varrho}{4}-\frac{k}{2}-\ell}\|u\|_{X(t)}^{p} .
$$

Using again (49), for the integral over $[t / 2, t]$ we obtain

$$
\begin{aligned}
\int_{t / 2}^{t}(1+t-s)^{-\frac{k}{2}-\ell}\|u(s, \cdot)\|_{L^{2 p}\left(\mathbf{H}_{n}\right)}^{p} \mathrm{~d} s & \lesssim \int_{t / 2}^{t}(1+t-s)^{-\frac{k}{2}-\ell}(1+s)^{-\frac{\varrho p}{2}+\frac{\varrho}{4}+\delta p\left(\frac{\varrho}{4}+\frac{1}{2}\right)} \mathrm{d} s\|u\|_{X(t)}^{p} \\
& \lesssim(1+t)^{-\frac{\varrho p}{2}+\frac{\varrho}{4}+\delta p\left(\frac{6}{4}+\frac{1}{2}\right)} \int_{t / 2}^{t}(1+t-s)^{-\frac{k}{2}-\ell} \mathrm{d} s\|u\|_{X(t)}^{p} \\
& \lesssim(1+t)^{-\frac{\varrho p}{2}+\frac{6}{4}+\delta p\left(\frac{6}{4}+\frac{1}{2}\right)-\frac{k}{2}-\ell+1}(\log (1+t))^{\ell}\|u\|_{X(t)}^{p} \\
& \lesssim(1+t)^{-\frac{\varrho}{4}-\frac{k}{2}-\ell}\|u\|_{X(t)}^{p} .
\end{aligned}
$$

Summarizing, from (48) we derived

$$
(1+t)^{\frac{6}{4}+\frac{k}{2}+\ell}\left\|\partial_{t}^{\ell} \nabla_{\mathrm{H}}^{k} u(t, \cdot)\right\|_{L^{2}\left(\mathbf{H}_{n}\right)} \lesssim \varepsilon_{0}+\|u\|_{X(t)}^{p} .
$$

Therefore, combining (47) and (50), it follows

$$
\|u\|_{X(T)} \lesssim \varepsilon_{0}+\varepsilon_{0}^{\frac{p+1}{2}}+\|u\|_{X(T)}^{\frac{p+1}{2}}+\|u\|_{X(T)}^{p}
$$

If $\varepsilon_{0}>0$ is small enough, then, from the last inequality we get that $\|u\|_{X(T)}$ is uniformly bounded, more precisely,

$$
\|u\|_{X(T)} \lesssim \varepsilon_{0}
$$

for any $T \in\left(0, T_{\max }\right)$ (cf. [16, Section 6$]$, for example). Besides, from

$$
\mathrm{e}^{\psi(t, \eta)} u(t, \eta)=\mathrm{e}^{\psi(t, \eta)} u_{0}(\eta)+\int_{0}^{t} \mathrm{e}^{\psi(t, \eta)} u_{t}(s, \eta) \mathrm{d} s
$$

and by using the monotonicity of $\psi$ with respect to $t$, we get

$$
\begin{aligned}
\left\|\mathrm{e}^{\psi(t, \cdot)} u(t, \cdot)\right\|_{L^{2}\left(\mathbf{H}_{n}\right)} & \lesssim \varepsilon_{0}+\int_{0}^{t}\left\|\mathrm{e}^{\psi(t, \cdot)} u_{t}(s, \cdot)\right\|_{L^{2}\left(\mathbf{H}_{n}\right)} \mathrm{d} s \lesssim \varepsilon_{0}+\int_{0}^{t}\left\|\mathrm{e}^{\psi(s, \cdot)} u_{t}(s, \cdot)\right\|_{L^{2}\left(\mathbf{H}_{n}\right)} \mathrm{d} s \\
& \lesssim \varepsilon_{0}(1+T),
\end{aligned}
$$

where in the last estimate we used (52). Therefore, if $T_{\max }<\infty$, then, it holds

$$
\limsup _{T \rightarrow T_{\max }^{-}}\left(\left\|\mathrm{e}^{\psi(t, \cdot)} u(t, \cdot)\right\|_{L^{2}\left(\mathbf{H}_{n}\right)}+\left\|\mathrm{e}^{\psi(t, \cdot)} \nabla_{\mathrm{H}} u(t, \cdot)\right\|_{L^{2}\left(\mathbf{H}_{n}\right)}\left\|\mathrm{e}^{\psi(t, \cdot)} u_{t}(t, \cdot)\right\|_{L^{2}\left(\mathbf{H}_{n}\right)}\right) \lesssim \varepsilon_{0}(1+T)<\infty .
$$

Nevertheless, this is impossible according to the last part of Theorem 2.1, so $T_{\max }=\infty$, that is $u$, has to be a global solution. The decay estimates for $u$ and its first order derivatives from the statement follows by the relation (52) which holds uniformly with respect to $T$. 


\section{Blow-up: proof of Theorem 2.3}

Before proving Theorem 2.3, we recall briefly the definition of weak solution to (1).

Definition 8.1. A weak solution of the Cauchy problem $(1)$ in $[0, T) \times \mathbf{H}_{n}$ is a function $u \in L_{\text {loc }}^{p}\left([0, T) \times \mathbf{H}_{n}\right)$ that satisfies

$$
\begin{gathered}
\int_{0}^{T} \int_{\mathbf{H}_{n}}|u(t, \eta)|^{p} \varphi(t, \eta) \mathrm{d} \eta \mathrm{d} t+\int_{\mathbf{H}_{n}}\left(u_{0}(\eta)+u_{1}(\eta)\right) \varphi(0, \eta) \mathrm{d} \eta-\int_{\mathbf{H}_{n}} u_{0}(\eta) \partial_{t} \varphi(0, \eta) \mathrm{d} \eta \\
=\int_{0}^{T} \int_{\mathbf{H}_{n}} u(t, \eta)\left(\partial_{t}^{2} \varphi(t, \eta)-\Delta_{\mathrm{H}} \varphi(t, \eta)-\partial_{t} \varphi(t, \eta)\right) \mathrm{d} \eta \mathrm{d} t
\end{gathered}
$$

for any $\varphi \in \mathscr{C}_{0}^{\infty}\left([0, T) \times \mathbf{H}_{n}\right)$. If $T=\infty$, we call $u$ a global in time weak solution to (1), else we call $u$ a local in time weak solution.

Proof of Theorem 2.3. We apply the so-called test function method. By contradiction, we assume that there exists a global in time weak solution $u$ to $(1)$.

Let us consider two bump functions $\alpha \in \mathscr{C}_{0}^{\infty}\left(\mathbb{R}^{n}\right)$ and $\beta \in \mathscr{C}_{0}^{\infty}(\mathbb{R})$. Furthermore, we require that $\alpha, \beta$ are radial symmetric and decreasing with respect to the radial variable, $\alpha=1$ on $B_{n}\left(\frac{1}{2}\right), \beta=1$ on $\left[-\frac{1}{4}, \frac{1}{4}\right], \operatorname{supp} \alpha \subset B_{n}(1)$ and $\operatorname{supp} \beta \subset(-1,1)$. If $R>1$ is a parameter, then, we define the test function $\varphi_{R} \in \mathscr{C}_{0}^{\infty}\left([0, \infty) \times \mathbb{R}^{2 n+1}\right)$ with separate variables as follows:

$$
\varphi_{R}(t, x, y, \tau) \doteq \beta\left(\frac{t}{R^{2}}\right) \alpha\left(\frac{x}{R}\right) \alpha\left(\frac{y}{R}\right) \beta\left(\frac{\tau}{R^{2}}\right) \quad \text { for any }(t, x, y, \tau) \in[0, \infty) \times \mathbb{R}^{2 n+1} .
$$

It is well-know that

$$
\left|\partial_{j} \alpha\right| \lesssim \alpha^{\frac{1}{p}} \quad \text { for any } 1 \leq j \leq n, \quad\left|\partial_{j} \partial_{k} \alpha\right| \lesssim \alpha^{\frac{1}{p}} \quad \text { for any } 1 \leq j, k \leq n, \quad\left|\beta^{\prime}\right| \lesssim \beta^{\frac{1}{p}}, \quad\left|\beta^{\prime \prime}\right| \lesssim \beta^{\frac{1}{p}} .
$$

Furthermore, $0 \leq \alpha, \beta \leq 1$ implies immediately $\alpha \leq \alpha^{\frac{1}{p}}$ and $\beta \leq \beta^{\frac{1}{p}}$. Therefore, from the relations

$$
\begin{aligned}
\partial_{t} \varphi_{R}(t, x, y, \tau)= & R^{-2} \beta^{\prime}\left(\frac{t}{R^{2}}\right) \alpha\left(\frac{x}{R}\right) \alpha\left(\frac{y}{R}\right) \beta\left(\frac{\tau}{R^{2}}\right) \\
\partial_{t}^{2} \varphi_{R}(t, x, y, \tau)= & R^{-4} \beta^{\prime \prime}\left(\frac{t}{R^{2}}\right) \alpha\left(\frac{x}{R}\right) \alpha\left(\frac{y}{R}\right) \beta\left(\frac{\tau}{R^{2}}\right) \\
\Delta_{\mathrm{H}} \varphi_{R}(t, x, y, \tau)= & R^{-2} \beta\left(\frac{t}{R^{2}}\right) \Delta \alpha\left(\frac{x}{R}\right) \alpha\left(\frac{y}{R}\right) \beta\left(\frac{\tau}{R^{2}}\right)+R^{-2} \beta\left(\frac{t}{R^{2}}\right) \alpha\left(\frac{x}{R}\right) \Delta \alpha\left(\frac{y}{R}\right) \beta\left(\frac{\tau}{R^{2}}\right) \\
& +R^{-3} \sum_{j=1}^{n} x_{j} \beta\left(\frac{t}{R^{2}}\right) \alpha\left(\frac{x}{R}\right) \partial_{j} \alpha\left(\frac{y}{R}\right) \beta^{\prime}\left(\frac{\tau}{R^{2}}\right)-R^{-3} \sum_{j=1}^{n} y_{j} \beta\left(\frac{t}{R^{2}}\right) \partial_{j} \alpha\left(\frac{x}{R}\right) \alpha\left(\frac{y}{R}\right) \beta^{\prime}\left(\frac{\tau}{R^{2}}\right) \\
& +\frac{1}{4} R^{-4}\left(|x|^{2}+|y|^{2}\right) \beta\left(\frac{t}{R^{2}}\right) \alpha\left(\frac{x}{R}\right) \alpha\left(\frac{y}{R}\right) \beta^{\prime \prime}\left(\frac{\tau}{R^{2}}\right)
\end{aligned}
$$

where $\Delta$ denotes the Laplace operator on $\mathbb{R}^{n}$, we get

$$
\begin{aligned}
\left|\partial_{t} \varphi_{R}\right| & \lesssim R^{-2}\left(\varphi_{R}\right)^{\frac{1}{p}} \\
\left|\partial_{t}^{2} \varphi_{R}\right| & \lesssim R^{-4}\left(\varphi_{R}\right)^{\frac{1}{p}} \lesssim R^{-2}\left(\varphi_{R}\right)^{\frac{1}{p}} \\
\left|\Delta_{\mathrm{H}} \varphi_{R}\right| & \lesssim R^{-2}\left(\varphi_{R}\right)^{\frac{1}{p}}
\end{aligned}
$$

We used that $\operatorname{supp} \varphi_{R} \subset\left[0, R^{2}\right] \times B^{n}(R) \times B^{n}(R) \times\left[-R^{2}, R^{2}\right]$ in order to estimate the polynomial terms in the estimate of $\left|\Delta_{\mathrm{H}} \varphi_{R}\right|$. 
Let us apply the definition of weak solution (53) to the test function $\varphi_{R}$. Hence, by (55) we obtain

$$
\begin{aligned}
\int_{0}^{\infty} \int_{\mathbf{H}_{n}} \mid & \left.u(t, \eta)\right|^{p} \varphi_{R}(t, \eta) \mathrm{d} \eta \mathrm{d} t+\int_{\mathbf{H}_{n}}\left(u_{0}(\eta)+u_{1}(\eta)\right) \varphi_{R}(0, \eta) \mathrm{d} \eta \\
& \leq \int_{0}^{\infty} \int_{\mathbf{H}_{n}}|u(t, \eta)|\left(\left|\partial_{t}^{2} \varphi_{R}(t, \eta)\right|+\left|\Delta_{\mathbf{H}} \varphi_{R}(t, \eta)\right|+\left|\partial_{t} \varphi_{R}(t, \eta)\right|\right) \mathrm{d} \eta \mathrm{d} t \\
& \lesssim R^{-2} \int_{0}^{\infty} \int_{\mathbf{H}_{n}}|u(t, \eta)|\left(\varphi_{R}(t, \eta)\right)^{\frac{1}{p}} \mathrm{~d} \eta \mathrm{d} t \\
& \leq R^{-2}\left(\int_{0}^{\infty} \int_{\mathbf{H}_{n}}|u(t, \eta)|^{p} \varphi_{R}(t, \eta) \mathrm{d} \eta \mathrm{d} t\right)^{\frac{1}{p}}\left(\iint_{\left[0, R^{2}\right] \times \mathscr{D}_{R}} \mathrm{~d} \eta \mathrm{d} t\right)^{\frac{1}{p^{\prime}}},
\end{aligned}
$$

where in the last step we used Hölder's inquality and the support property for $\varphi_{R}$. Let us introduce now the $R$-dependent integrals

$$
I_{R} \doteq \int_{0}^{\infty} \int_{\mathbf{H}_{n}}|u(t, \eta)|^{p} \varphi_{R}(t, \eta) \mathrm{d} \eta \mathrm{d} t, \quad J_{R} \doteq \int_{\mathbf{H}_{n}}\left(u_{0}(\eta)+u_{1}(\eta)\right) \varphi_{R}(0, \eta) \mathrm{d} \eta .
$$

Due to the assumption on the data in (9), we have $\liminf _{R \rightarrow \infty} J_{R}>0$, which implies in turn that $J_{R}>0$ for $R \geq R_{0}$, where $R_{0}$ is a suitable positive real number. Indeed, from $\operatorname{supp} \varphi_{R}(0, \cdot) \subset \mathscr{D}_{R}$ and $\varphi_{R}(0, \cdot)=1$ on $\mathscr{D}_{R / 2}$ we get trivially

$$
J_{R}=\int_{\mathscr{D}_{R}}\left(u_{0}(\eta)+u_{1}(\eta)\right) \varphi_{R}(0, \eta) \mathrm{d} \eta \geq \int_{\mathscr{D}_{R / 2}}\left(u_{0}(\eta)+u_{1}(\eta)\right) \mathrm{d} \eta .
$$

Then, for $R \geq R_{0}$ the estimate in (56) yields

$$
I_{R} \leq I_{R}+J_{R} \lesssim R^{-2+\frac{2 n+4}{p^{\prime}}} I_{R}^{\frac{1}{p}}=R^{Q-\frac{Q+2}{p}} I_{R}^{\frac{1}{p}}
$$

where we applied meas $\left(\mathscr{D}_{R}\right) \approx R^{Q}$. When the exponent of $R$ in the right-hand side of the last inequality is negative, i.e., for $p<p_{\text {Fuj }}(\mathbb{Q})$, we have that

$$
0 \leq I_{R}^{1-\frac{1}{p}} \lesssim R^{Q-\frac{Q+2}{p}} \longrightarrow 0 \quad \text { as } R \rightarrow \infty .
$$

Thus, $\lim _{R \rightarrow \infty} I_{R}=0$. However, this is not possible, because the term $J_{R}$ is positive for $R$ sufficiently large. So, letting $R \rightarrow \infty$ in (58) we find the contradiction we were looking for. In order to get a contradiction in the critical case $p=p_{\text {Fuj }}(\mathbb{Q})$ too, we need to refine the estimate in (56). Indeed, we can use the fact that $\partial_{t} \varphi_{R}$ is supported in $\widehat{\mathscr{P}}_{R} \doteq\left[\frac{R^{2}}{4}, R^{2}\right] \times \mathscr{D}_{R}$ and $\Delta_{\mathrm{H}} \varphi_{R}$ is supported in $\widetilde{\mathscr{P}}_{R} \doteq\left[0, R^{2}\right] \times\left(\mathscr{D}_{1, R} \cup \mathscr{D}_{2, R} \cup \mathscr{D}_{3, R}\right)$, where

$$
\begin{aligned}
& \mathscr{D}_{1, R} \doteq\left(B_{n}(R) \backslash B_{n}(R / 2)\right) \times B_{n}(R) \times\left[-R^{2}, R^{2}\right], \\
& \mathscr{D}_{2, R} \doteq B_{n}(R) \times\left(B_{n}(R) \backslash B_{n}(R / 2)\right) \times\left[-R^{2}, R^{2}\right], \\
& \mathscr{D}_{3, R} \doteq B_{n}(R) \times\left(B_{n}(R)\right) \times\left(\left[-R^{2}, R^{2}\right] \backslash\left[-R^{2} / 4, R^{2} / 4\right]\right) .
\end{aligned}
$$

Consequently, for $R \geq R_{0}$ we may improve (56) as follows

$$
I_{R} \leq I_{R}+J_{R} \lesssim \widehat{I}_{R}^{\frac{1}{p}}+\widetilde{I}_{R}^{\frac{1}{p}}
$$

where

$$
\widehat{I}_{R} \doteq \iint_{\widehat{\mathscr{P}}_{R}}|u(t, \eta)|^{p} \varphi_{R}(t, \eta) \mathrm{d} \eta \mathrm{d} t \quad \text { and } \quad \widetilde{I}_{R} \doteq \iint_{\widetilde{P}_{R}}|u(t, \eta)|^{p} \varphi_{R}(t, \eta) \mathrm{d} \eta \mathrm{d} t .
$$


In the critical case $p=p_{\text {Fuj }}(\mathbb{Q})$, from (58) it follows that $I_{R}$ is uniformly bounded as $R \rightarrow \infty$. Using the monotone convergence theorem, we find

$$
\lim _{R \rightarrow \infty} I_{R}=\lim _{R \rightarrow \infty} \int_{0}^{\infty} \int_{\mathbf{H}_{n}}|u(t, \eta)|^{p} \varphi_{R}(t, \eta) \mathrm{d} \eta \mathrm{d} t=\int_{0}^{\infty} \int_{\mathbf{H}_{n}}|u(t, \eta)|^{p} \mathrm{~d} \eta \mathrm{d} t \lesssim 1 .
$$

This means that $u \in L^{p}\left([0, \infty) \times \mathbf{H}_{n}\right)$. Applying now the dominated convergence theorem, as the characteristic functions of the sets $\widehat{\mathscr{P}}_{R}$ and $\widetilde{\mathscr{P}}_{R}$ converge to the zero function for $R \rightarrow \infty$, we have

$$
\begin{aligned}
& \lim _{R \rightarrow \infty} \widehat{I}_{R}=\lim _{R \rightarrow \infty} \iint_{\widehat{\mathscr{P}}_{R}}|u(t, \eta)|^{p} \varphi_{R}(t, \eta) \mathrm{d} \eta \mathrm{d} t=0, \\
& \lim _{R \rightarrow \infty} \widetilde{I}_{R}=\lim _{R \rightarrow \infty} \iint_{\widetilde{\mathscr{P}}_{R}}|u(t, \eta)|^{p} \varphi_{R}(t, \eta) \mathrm{d} \eta \mathrm{d} t=0 .
\end{aligned}
$$

Also, letting $R \rightarrow \infty$, (59) implies $\lim _{R \rightarrow \infty} I_{R}=0$ which provides the desired contradiction in turn, as we have already seen in the subcritical case. The proof is completed.

\section{Acknowledgments}

V. Georgiev is supported in part by GNAMPA - Gruppo Nazionale per l'Analisi Matematica, la Probabilità e le loro Applicazioni, by Institute of Mathematics and Informatics, Bulgarian Academy of Sciences, by Top Global University Project, Waseda University and by the University of Pisa, Project PRA 201849. A. Palmieri is supported by the University of Pisa, Project PRA 201849.

\section{References}

[1] Bahouri H, Chemin J-Y, Xu C-J, Trace and trace lifting theorems in weighted Sobolev spaces, Journal of the Inst. of Math. Jussieu 4(4) (2005), 509-552.

[2] Bahouri H, Chemin J-Y, Xu C-J, Trace theorem on the Heisenberg group, Ann. Inst. Fourier, Grenoble 59(2) (2009), 491-514.

[3] Berhanu S, Pesenson I, The trace problem for vector fields satisfying HörmanderâẮ́s condition, Math. Z. 231 (1999), 103-122.

[4] Bihari I, A generalization of a lemma of Bellman and its application to uniqueness problems of differential equations, Acta Math. Acad. Sci. Hungar. 7(1) (1956), 81-94.

[5] Chen J, Rocha E M, A Class of Sub-elliptic Equations on the Heisenberg Group and Related Interpolation Inequalities, in: Advances in Harmonic Analysis and Operator Theory, in: Oper. Theory Adv. Appl., vol. 229, Birkhäuser/Springer, Basel AG, Basel, 2013, 123-137.

[6] Chen W, Palmieri A, Weakly coupled system of semilinear wave equations with distinct scale-invariant terms in the linear part, Z. Angew. Math. Phys. 70(2) (2019) 70: 67. https://doi.org/10.1007/s00033-019-1112-4

[7] D'Abbicco M, The threshold of effective damping for semilinear wave equation, Math. Meth. Appl. Sci. 38 (2015), $1032-1045$.

[8] D'Abbicco M, Lucente S, Reissig M, Semi-linear wave equations with effective damping, Chinese Annals of Mathematics, Ser. B, 34 (2013), 345-380.

[9] Ebert M R, Reissig M, Methods for partial differential equations. Qualitative properties of solutions, phase space analysis, semilinear models, Birkhäuser, Basel, 2018

[10] Georgiev V, Palmieri A, Lifespan estimates for local in time solutions to the semilinear heat equation on the Heisenberg group, preprint, arxiv:1905.05696 (2019).

[11] Georgiev V, Palmieri A, Upper bound estimates for local in time solutions to the semilinear heat equation on stratified Lie groups in the sub-Fujita case, preprint.

[12] Ikehata R, Tanizawa K, Global existence of solutions for semilinear damped wave equations in $\mathbb{R}^{N}$ with noncompactly supported initial data, Nonlinear Anal. 61(7) (2005), 1189-1208.

[13] Matsumura A, On the asymptotic behavior of solutions of semi-linear wave equations, Publ. Res. Inst. Math. Sci. 12(1) $(1976 / 77), 169-189$.

[14] Mitidieri E, Pohozaev S, A priori estimates and the absence of solutions of nonlinear partial differential equations and inequalities, Proc. Steklov Inst. Math. 234 (2001), 1-362.

[15] Nunes do Nascimento W, Palmieri A, Reissig M, Semi-linear wave models with power non-linearity and scale-invariant time-dependent mass and dissipation, Math. Nachr. 290(11/12) (2017), 1779-1805.

[16] Palmieri A, Global existence of solutions for semi-linear wave equation with scale-invariant damping and mass in exponentially weighted spaces, J. Math. Anal. Appl. 461(2) (2018), 1215-1240. 
[17] Palmieri A, Decay estimates for the linear damped wave equation on the Heisenberg group, preprint, arxiv:1908.02657 (2019).

[18] Pesenson I, The trace problem and Hardy operator for non-isotropic function spaces on the Heisenberg group, Comm. Partial Differential Equations, 19(3/4) (1994), 655-676 .

[19] Ruzhansky M, Tokmagambetov N, Nonlinear damped wave equations for the sub-Laplacian on the Heisenberg group and for Rockland operators on graded Lie groups J. Differential Equations 265 (2018), 5212-5236.

[20] Ruzhansky M, Yessirkegenov N, Existence and non-existence of global solutions for semilinear heat equations and inequalities on sub-Riemannian manifolds, and Fujita exponent on unimodular Lie groups, preprint, arxiv:1812.01933v2 (2018).

[21] Todorova G, Yordanov B, Critical exponent for a nonlinear wave equation with damping, J. Differential Equations 174(2) (2001), 464-489. 